\title{
Order-Disorder Competitive Cooperation in Equiatomic 3d-Transition-Metal Quaternary Alloys: Phase Stability and Electronic Structure
}

\author{
Hiroshi Mizuseki \\ Korea Institute of Science and Technology (KIST), Seoul 02792, Republic of Korea, \\ Ryoji Sahara \\ National Institute for Materials Science (NIMS), Tsukuba 305-0047, Japan, \\ Kenta Hongo* \\ Research Center for Advanced Computing Infrastructure, \\ JAIST, Asahidai 1-1, Nomi, Ishikawa 923-1292, Japan
}

(Dated: January 3, 2022)

\begin{abstract}
We use high-throughput first-principles sampling to investigate competitive factors that determine the crystal structure of high-entropy alloys (HEAs) and the energetics dependence of the stable phase on the atomic configuration of fully ordered $\mathrm{L}_{2}, \mathrm{D}_{22}$, and random solid solution (RSS) phases of equiatomic quaternary alloys comprising four of the six constituent elements $(\mathrm{Cr}, \mathrm{Mn}, \mathrm{Fe}, \mathrm{Co}, \mathrm{Ni}$, and $\mathrm{Cu})$. Considering the configurational entropy, we demonstrate that valence electron concentration (VEC) and temperature are crucial to determine the phase stability of HEAs at finite temperatures, wherein the ordered phases are energetically more favorable than RSS phases. Some $\mathrm{D0}_{22}$ phases with high VEC are energetically more stable than $\mathrm{L}_{2}$ phases, though both phases are metastable. Further, we explore magnetic configurations to identify the origin of the enthalpy term. The calculations reveal that ordered phases comprising antiferromagnetic atoms surrounded by ferromagnetic atoms are energetically stable. The quantitative structure-property relationship is also discussed.
\end{abstract}

\section{INTRODUCTION}

High-entropy alloys (HEAs) [1, 2] have been gaining increasing attention because of their novel material properties that cater to a wide range of applications. Although HEAs were originally applied to structural materials that required both strength and ductility, [3-7] their recent applications have been extended to other functional materials such as catalysis, [8] superconductors, [9] radiation resistance materials [10], and magnetic materials. [11, 12] These extensions of their applications represent a new direction of materials design based on the fundamental and key concept of "high-entropy." Along with this new direction, the comprehensive understanding of quantitative structure-property relationship (QSPR) is crucial for exploiting their potential applications. However, its most fundamental informationmicroscopic/atomistic crystal structures of HEAs-remains unknown [13]; this implies that the atomistic characterization of HEA structures is challenging for both "experiments" and "theories/computations," in terms of "spatial resolutions" [14] and "computational costs for vast amounts of possible atomic configurations," [15, 16] respectively.

It is considered that HEAs have random solid solutions (RSSs) instead of ordered phases. Although disordered face-/body-centered cubic (FCC/BCC) phases (A1/A2) have been reported as single HEA phases, [17] ordered FCC/BCC $\left(\mathrm{L}_{2} / \mathrm{B} 2\right)$ and hexagonal close-packed (HCP, A3) have also been found. [18-20] Although there are a few examples of ordered phases, perfect randomness in some types of HEAs has been excluded in several studies. For example, short range order (SRO) in HEAs has been reported [21-23]; however, the existence of long-range order (LRO) is rarely discussed and remains a controversial topic. [24, 25] The most famous ordered phase in HEAs is $\mathrm{CrFeCoNi}$, with the $\mathrm{L}_{2}$ structure, [25] wherein the antiferromagnetic $\mathrm{Cr}$ atoms occupy the ordered lattice sites surrounded by randomly distributed ferromagnetic $\mathrm{Ni}, \mathrm{Fe}$, and $\mathrm{Co}$ atoms. This can be interpreted as a spin-driven stabilization of the atomic configuration, which indicates that enthalpy enhancement by magnetization cooperates partial randomness and then overcomes the entropy because of perfect randomness. This was theoretically predicted [25-27] and verified experimentally only for the "surface" using scanning transmission electron microscopy (STEM) [25]; experimental verifications for the "bulk" have not been feasible because the constituent atoms are adjoining elements in the periodic table and have similar chemical properties and comparable radii, which makes them indistinguishable when using spectroscopic methods. [14] Thus, the question arises if the spin-driven stabilization is unique for $\mathrm{Cr}$ FeCoNi or if it generally holds for other quaternary alloys. To address this issue from a microscopic perspective, instead of experiments, first-principles approaches are the most relevant for identifying atomistic structures of HEAs and elucidating their QSPR [28-31], though the previous studies dealt with only single composites.

We systematically focus on 3d-transition-metal-based quaternary alloys with $\mathrm{Cr}, \mathrm{Mn}, \mathrm{Fe}, \mathrm{Co}, \mathrm{Ni}$, and $\mathrm{Cu}$ as the constituent elements; a total of $15\left(=\left(\begin{array}{l}6 \\ 4\end{array}\right)=: \frac{6 !}{4 ! 2 !}\right)$ different types of composites are considered (TABLE【). These elements are most commonly used magnetic HEA constituents [19], and all quaternary alloy combinations that results from them can be speculated to have the FCC phase [32]. Therefore, they are expected to be disordered/RSSs as their atomic sizes and the nature of chemical bonds formed by them are similar. Our first-principles study revealed that differences in their magnetic properties with respect to $\mathrm{CrFeCoNi}$ lead to different magnetic orderings: our target phases were not restricted to 
only RSSs and $\mathrm{L}_{2}$ ordered phases; they extended to the $\mathrm{D}_{22}$ ordered phases as well (Figure 1), motivated by well-known studies on order-disorder phase competition in ordered alloys [33-38]. The $\mathrm{L}_{2}$ and $\mathrm{D}_{22}$ phases are ordered crystal structures for 25:75 at.\% FCC alloys. As prototype systems for ordered phases, their phase competition has been well investigated for conventional alloys in terms of valence electron concentration (VEC). [33, 34] For example, the $\mathrm{L}_{2}$ phase of intermetallic compounds is observed for VEC $\geq 7.5$. However, to the best of our knowledge, there is no previously reported study on their counterpart for HEAs; in HEAs, VEC values are known to correlate well with structure types (FCC/BCC structures prefer higher/lower VEC values). [3942] Thus, we analyze the RSS- $\mathrm{L}_{2}-\mathrm{D}_{22}$ thermodynamical competition in the 3d-transition-metal quaternary system in terms of VEC.

We performed high-throughput first-principles sampling from four types of $\mathrm{L}_{2}$ and $\mathrm{D}_{22}$ phases to understand the RSS- $\mathrm{L}_{2}-\mathrm{D} 0_{22}$ competition and their QSPR, and from one type of RSS phase for 15 different composites. We found that composites with lower VEC values tend to have ordered phases over wide temperature ranges; however, for higher VEC values, RSSs emerge as the temperature increases. In addition, we discuss the distribution of the magnetic moment at the first nearest neighbor site and the constituent elements in our HEAs. We believe that phase stability is realized by order-disorder "competitive cooperation" rather than by the "commonly conceived" order-disorder competition: the former is caused by the cooperation between a "magnetically ordered atomic configuration composed of one element gaining enthalpy" and "fully disordered/random configuration composed of the other three elements gaining entropy," which we propose in this study; the latter is the realization of either "fully ordered configuration" or "fully random configuration." Their difference is not sufficiently appreciated in the HEA community.

The paper is organized as follows: Computational details in the present study are given in "METHODS". In "RESULTS", we start with our main finding "Order-Disorder Competitive Cooperation", followed by "Energetics" and "Magnetic ordering", which can be explained by "Quantitative Structure-Property Relationship". "SUMMARY AND FUTURE PROSPECTS" summarize our findings and their significance towards further investigation in HEAs.

\section{METHODS}

\section{A. HEA structural models}

HEAs have been regarded as solid solutions, wherein every site in the crystal structure is randomly occupied by the constituent elements. However, it is difficult to reproduce such a random configuration with a small simulation cell size. Therefore, finding an appropriate method to mimic the configuration of HEAs under a limited periodic condition is crucial for theoretical investigations. This problem can be solved by evaluating the correlation functions of the atomic configurations in HEAs. For instance, Zunger et al. proposed the concept of special quasirandom structures (SQSs) [43], which are supercell approximations for a disordered system optimized to mimic the random local atomic environment. Therefore, the SQS approach is widely applied to HEA research because it provides a good approximation for the RSS phase. However, it is difficult to discuss the energetics and phase stability of HEAs in detail using only one configuration obtained from the SQS model. It has previously been reported that applying several SQS configurations of HEAs leads to non-negligible energy fluctuations $(0.01-0.02 \mathrm{eV} /$ atom). [1, 44] We performed high-throughput first-principles sampling to provide insights regarding the phase stability of HEAs with high accuracy and reliability for overcoming the disadvantage of using a single configuration in the SQS approach.

Two-, three-, and four-body clusters were selected for a correlation function, with the Warren-Cowley short-range order (SRO) parameter evaluated by homogeneous and heterogeneous elements [45, 46]. The range of the SRO parameters was restricted to the fourth nearest-neighbor site for two-body clusters and to the third nearest-neighbor site for three- and four-body clusters to reduce the computational time. For the RSS phase, the configurations of four elements were explored to realize a better correlation function using the Metropolis algorithm [47] with a wide range of fictitious temperatures. For both the ordered and RSS phases, four elements were considered for evaluating the correlation function. In the $\mathrm{L}_{2}$ ordered phase, one element was fixed at the cubic corner (CC) sites in the FCC lattice, while the other three elements in the quaternary system occupied the face-centered sites to realize an ideal $\mathrm{L} 1_{2}$ ordered phase with a good correlation function. The $\mathrm{D}_{22}$ phase was also constructed in a similar manner. A wide range of occupancy rates was used to elucidate the influence of the number of first nearest-neighbor sites on the formation energy. Accordingly, a large number of atomic configurations were randomly generated for RSS structures (Fig. 6. Our structural models of the quaternary alloys were described by $2 \times 2 \times 2$ supercells with 32 sites or $4 \times 4 \times 4$ supercells with 256 sites, depending on the computed quantities; the equiatomic system comprises 8 atoms in the $2 \times 2 \times 2$ supercell and 64 atoms in the $4 \times 4 \times 4$ supercell for each element. The crystal structures were drawn using the VESTA software [48].

\section{B. High-throughput first-principles sampling}

The free energy of formation (Figure 4 and magnetic moment (Figure 5) for each phase were obtained based on 100 configurations using a $2 \times 2 \times 2$ supercell. Figure S- 2 shows the formation free energies obtained using 100 configurations. The dependences of the formation energy on the occupancy rate (Figure 6) and the bond-length distributions (Figure 7 ) for the $\mathrm{CrFeCoNi}$ quaternary alloy were obtained based on 1,500 and 100 configurations, respectively, using a $4 \times 4 \times 4$ supercell.

We carried out high-throughput spin-polarized firstprinciples simulations based on DFT [49] using the Vienna ab initio simulation package (VASP) by inputing all gener- 
ated HEA models above [50, 51]. The PBEsol functional [52] was selected for the electronic structure calculations and geometry relaxation. Projector-augmented wave (PAW) [53, 54] potentials were used to consider the interactions between the ion cores and valence electrons. The Brillouin zone was described by a set of k-points in a $3 \times 3 \times 3$ grid mesh using the Monkhorst-Pack method. [55] For structural optimization, the energy convergence criterion for electronic iterations was set at $10^{-2} \mathrm{eV} / \AA$. The lattice parameter was set at $3.495 \AA$ during structural optimization to reduce the calculation time. As an initial condition, $\mathrm{Fe}, \mathrm{Co}, \mathrm{Ni}$, and $\mathrm{Cu}$ were set as ferromagnetic, whereas $\mathrm{Cr}$ was set as antiferromagnetic. For systems without $\mathrm{Cr}, \mathrm{Mn}$ was set as antiferromagnetic; otherwise, $\mathrm{Mn}$ was set as ferromagnetic as an initial condition because its magnetism is affected by $\mathrm{Cr}$.

\section{Formation energy and formation free energy}

To discuss phase stability, the formation energy $E_{f}(\mathrm{HEA})$ is defined as

$$
E_{f}(\mathrm{HEA})=E(\mathrm{HEA})-\sum_{i} x_{i} E\left(X_{i}\right)
$$

Here, $E$ (HEA) denotes the total energy per atom of the HEA with the alloying elements; $X_{i}$ and $x_{i}$ represent the fractions of the alloying elements; and $E\left(X_{i}\right)$ denote the energies per atom of the alloying elements $X_{i}$ in their ground state structures, i.e., BCC Cr, $I \overline{4} 3 m \mathrm{Mn}, \mathrm{BCC} \mathrm{Fe}, \mathrm{HCP} \mathrm{Co}, \mathrm{FCC} \mathrm{Ni}$, and FCC $\mathrm{Cu}$, which are estimated by referring to the Open Quantum Materials Database [56, 57]. A negative value of the formation energy implies that the HEA is stable, whereas a positive value implies that it is less stable than the ground states of the pure alloying elements.

Temperature is one of the most important factors that governs the phase stability of materials because most materials are used in a finite temperature range instead of being used at absolute zero. [58]. Here, the Helmholtz free energy is defined as

$$
\begin{aligned}
F(V, T) & =E_{\text {el }}(V)+E_{\text {vib }}(V, T) \\
& -T S_{\text {ele }}(V, T)-T S_{\mathrm{vib}}(V, T)-T S_{\text {config }}(V, T),
\end{aligned}
$$

where $E_{\mathrm{el}}(V)$ denotes the internal energy, and the phonon vibration contribution includes the lattice vibration energy, i.e., $E_{\text {vib }}(V, T)$, and lattice vibration entropy, i.e., $S_{\text {vib }}(V, T)$. Further, $S_{\text {config }}(V, T)$ represents the atomic configurational entropy, and the last term $S_{\text {ele }}(V, T)$ denotes the electronic-scale entropy contribution, which includes thermal excitation and spin polarization. In this study, contributions from the vibrational and electronic entropy terms are not included owing to the limitations of the calculations. [59] Further, the vibrational contributions for both the ordered and RSS phases were estimated and found to be comparable [60]. The contributions of the electronic and magnetic terms are smaller than that of the configurational term [61]. $S_{\text {config }}$ in the configurational entropy term is given by $S_{\text {config }}=R \sum_{i} c_{i} \ln c_{i}$, where $c_{i}$ denotes the ratio between the number of atoms of a disordered component and the total number of disordered atoms, and $R$ represents the gas constant. In this study, we introduced an equiatomic quaternary system and calculated $S_{\text {config }}=R \ln (3) \times 0.75=0.824 R$ for the ordered phases and $S_{\text {config }}=R \ln (4)=1.386 R$ for the RSS phase. In other words, the RSS phase has a greater configurational entropy contribution. Therefore, the free energy of formation is calculated by

$$
F_{f}(\mathrm{HEA})=E(\mathrm{HEA})-T S_{\text {config }} .
$$

\section{RESULTS}

\section{A. Order-Disorder Competitive Cooperation}

One of the main objectives of this study is to investigate whether an ordered phase $\left(\mathrm{OP}_{0}\right)$ is more stabilized than the corresponding RSS for each studied quaternary alloy. That is, we intended to determine the "crossover" temperature $\left(T_{c}\right)$ at which the phase transition between the $\mathrm{OP}_{0}$ and RSS occurs for each composite of the quaternary alloy. Therefore, it is important to consider the configurational entropy term to discuss phase stability in HEAs in the finite temperature region. It is imperative to quantitatively analyze the influences of VEC and temperature on the stable HEA crystal structures for investigating the order-disorder competitive cooperation and phase stability. This is because the enthalpy term defined at the absolute zero temperature correlates with VEC, whereas the entropy effect is temperature dependent; the most stable phase is determined by free energy, i.e., the sum of the enthalpy and entropy terms. Since the direct elucidation of QSPR is difficult, material features (or descriptors) such as VEC [39, 40] are "inserted" between "structure" and "property"; then, two step analyses are conducted based on "structure-feature" and "feature-property" relationships. Guo et al. [39, 40] reported several HEA systems wherein the single FCC phase was stable in the high VEC region (VEC $>$ 8.0), the single $\mathrm{BCC}$ phase was stable in the low VEC region (VEC < 6.87), and the mixed FCC and BCC phases appeared in the intermediate region $(6.87<\mathrm{VEC}<8.0)$. BCC and FCC enhance the strength and ductility, respectively [1]. The average VEC values of magnetic HEAs are greater than 7.5 , and therefore, they possess the FCC structure. Hence, this study does not focus on the lattice type of magnetic HEAs, but on their magnetic orderings. We investigate the suitability of VEC to identify the orderings, which would be the first step toward successful designing HEAs.

TABLE【lists the quaternary alloy composites investigated in this study (Composite), average VECs ( $\mathrm{VEC}_{\mathrm{ave}}$ ), average melting temperature of the four constituent elements in the alloy $\left(\bar{T}_{m}\right)$, ordered phase with the lowest energy at $0 \mathrm{~K}$ $\left(\mathrm{OP}_{0}\right)$, temperature at which stability is attained for each phase $\left(T_{s}(X)\left(X=\mathrm{L1}_{2}, \mathrm{D}_{22}, \mathrm{RSS}\right)\right)$, "crossover" temperature between the ordered phase $\mathrm{OP}_{0}$ and RSS $\left(T_{c}\right)$, and the corresponding free energy of formation at $T_{c}\left(F_{\mathrm{c}}\right)$. The the most stable solid phase (MSSP) of each composite below $\bar{T}_{m}$ and the corresponding temperature range $\left(\Delta T_{s}\right)$ are also listed in 
the table. The free energy of formation considering the configurational entropy contribution is elucidated in Eq. (2). To estimate estimating the free energy of formation, we can consider the ordered phases as a 75 at.\% equiatomic ternary alloy for the configurational entropy term because one of the elements is completely located at a specific site (Fig. 11 (a) or (b)).

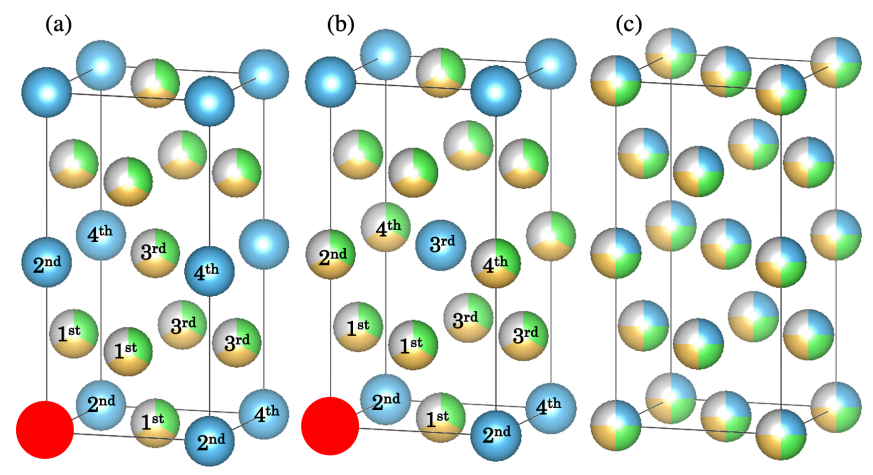

FIG. 1: Crystal structures of (a) $\mathrm{L1}_{2}\left(\mathrm{Cu}_{3} \mathrm{Au}\right.$-type, space group $P m \overline{3} m$, no. 221), (b) $\mathrm{D0}_{22}\left(\mathrm{Al}_{3}\right.$ Ti-type, space group I4/mmm, no. 139), and (c) RSS for quaternary alloys. Labels indicate the first, second, third, and fourth nearest neighbor sites from the red site located at the cubic corner.

Consequently, one element of the ordered phases can be ignored for the number of the elements considering the configurational entropy term. Alternatively, the RSS phases need to be treated as a quaternary alloy. Therefore, the contribution of the configurational entropy term for the RSS phase is larger than that for the ordered phases. Accordingly, the slope of the free energy of formation for the RSS phase is steeper than that for the ordered phases. The ordered phases consequently appear gradually as the temperature increases, and this is followed by the appearance of the RSS phases at higher temperatures. The above discussion presupposes thermal equilibrium. Note that ordered phases that thermally equilibrate at higher temperatures can appear even at ambient temperatures by non-equilibrium processing, i.e., rapid solidification and cooling. [62]

Figure 2 shows the free energy of formation of the CrMnCoNi quaternary alloy as a function of temperature. The temperature dependence of the free energy of formation of the other alloys, including the CrMnCoNi alloy, is shown in Fig. S-1 (supplementary information). For the both ordered phases, $\mathrm{Cr}-\mathrm{L}_{2}$ and $\mathrm{Cr}-\mathrm{DO}_{22}$ are the most stable phase of each ordered phase (Fig. 4(3)). Therefore, we compare the free energies of formation of the $\mathrm{Cr}-\mathrm{L}_{2}$ and $\mathrm{Cr}-\mathrm{DO}_{22}$ ordered phases and the RSS phase up to $\bar{T}_{m}(1799 \mathrm{~K})$. The temperature dependence can be seen as follows:

1. Within the low-temperature region (up to $282 \mathrm{~K}$ ), all ordered and RSS phases exhibit a positive free energy of formation, and they are less stable than the corresponding ground state of each pure element.

2. At $282-1003 \mathrm{~K}, \mathrm{Cr}-\mathrm{L}_{2}$ shows a negative free energy of formation, which is lower than those of the $\mathrm{Cr}-\mathrm{DO}_{22}$ and RSS phases. Therefore, $\mathrm{Cr}-\mathrm{L}_{2}$ is the most stable phase in this temperature region.

3. Beyond $1003 \mathrm{~K}$, the RSS phase shows a lower free energy of formation than $\mathrm{Cr}-\mathrm{L}_{2}$ and becomes a stable phase in the high-temperature region.

Meanwhile, the $\mathrm{Cr}-\mathrm{DO}_{22}$ phase does not appear as the most stable phase in the entire temperature range. Consequently, in the case of the CrMnCoNi quaternary alloy $\left(\mathrm{VEC}_{\mathrm{ave}}=8.00\right)$, the $\mathrm{L}_{2}$ crystal structure appears as an ordered phase in a specific temperature region.

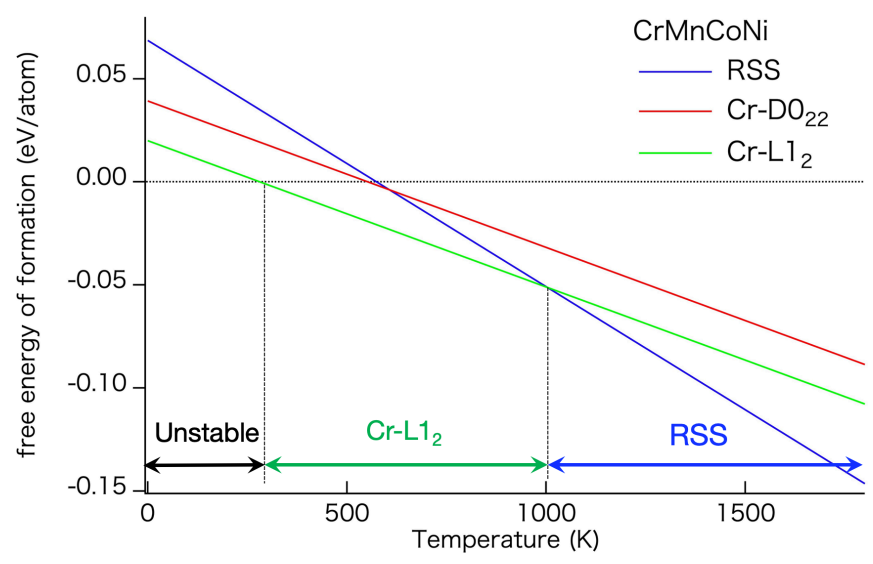

FIG. 2: Free energy of formation of CrMnCoNi quaternary alloy as a function of temperature up to its melting temperature $(1799 \mathrm{~K})$. Blue, red, and green lines indicate the $\mathrm{RSS}, \mathrm{Cr}-\mathrm{D0}_{22}$, and $\mathrm{Cr}-\mathrm{L}_{2}$ ordered phases, respectively.

Further, as shown in Fig. S-1 and TABLE[, the CrMnFeCo $\left(1 ; \mathrm{VEC}_{\mathrm{ave}}=7.5\right), \mathrm{CrMnFeNi}\left(2 ; \mathrm{VEC}_{\mathrm{ave}}=7.75\right), \mathrm{CrFe}-$ CoNi (5; $\left.\mathrm{VEC}_{\mathrm{ave}}=8.25\right), \operatorname{MnFeCoNi}\left(9 ; \mathrm{VEC}_{\mathrm{ave}}=8.5\right)$, and $\mathrm{FeCoNiCu}\left(15 ; \mathrm{VEC}_{\mathrm{ave}}=9.5\right)$ quaternary alloys exhibit the same phase transition between the $\mathrm{L}_{2}$ ordered phase and RSS phase at a specific temperature. However, the $\mathrm{CrFe}$ CoNi (5; $\left.\operatorname{VEC}_{\mathrm{ave}}=8.25\right)$ and $\operatorname{MnFeCoNi}\left(9 ; \mathrm{VEC}_{\mathrm{ave}}=8.5\right)$ quaternary alloys exhibited the $\mathrm{L}_{2}$ structure at $0 \mathrm{~K}$. Furthermore, the $\mathrm{CrMnCoCu}\left(6 ; \mathrm{VEC}_{\text {ave }}=8.25\right), \mathrm{CrFeCoCu}(7$; $\left.\mathrm{VEC}_{\mathrm{ave}}=8.5\right), \mathrm{CrMnNiCu}\left(8 ; \mathrm{VEC}_{\mathrm{ave}}=8.5\right), \mathrm{CrFeNiCu}$ $\left(10 ; \mathrm{VEC}_{\mathrm{ave}}=8.75\right), \mathrm{MnFeCoCu}\left(11 ; \mathrm{VEC}_{\mathrm{ave}}=8.75\right), \mathrm{Cr}-$ $\mathrm{CoNiCu}\left(12 ; \mathrm{VEC}_{\mathrm{ave}}=9.0\right), \operatorname{MnFeNiCu}\left(13 ; \mathrm{VEC}_{\mathrm{ave}}=9.0\right)$, and $\mathrm{MnCoNiCu}\left(14 ; \mathrm{VEC}_{\mathrm{ave}}=9.25\right)$, quaternary alloys show the RSS phase only below the melting temperature because of the small difference in the free energy of formation at $0 \mathrm{~K}$. (Fig. S1 (6)-(8), and (9)-(14)) The CrMnFeCu (4; $\mathrm{VEC}_{\mathrm{ave}}=$ 8.0) quaternary alloy is unstable in the entire temperature range.

The results of this study are summarized as follows: The alloys without $\mathrm{Cu}$ as a constituent prefer the $\mathrm{L}_{2}$ ordered phases for small VEC values $(\leq 8.25)$. The Mn-based $\mathrm{L}_{2}$ of $\mathrm{MnFe}$ $\mathrm{CoNi}(9)$ and the Fe-based $\mathrm{L}_{2}$ of $\mathrm{FeCoNiCu}(15)$ are also stable under a specific temperature. The free energy of formation of the $\mathrm{DO}_{22}$ ordered phases are lower than $\mathrm{L}_{2}$ ordered phases 
TABLE I: Temperature dependence of the most stable structures for 15 cases of equiatomic quaternary systems (column name:

"Composite") investigated in the present study. $\mathrm{VEC}_{\mathrm{ave}}$ corresponds to the average valence electron concentration of the composite, where $\mathrm{Cr}, \mathrm{Mn}, \mathrm{Fe}, \mathrm{Co}, \mathrm{Ni}$, and $\mathrm{Cu}$ have VEC values of $6,7,8,9,10$, and 11, respectively. $\bar{T}_{m}$ represents the average melting temperature of the four elements that comprise the composite. $\mathrm{OP}_{0}$ represents the ordered phase possessing the lowest energy at $0 \mathrm{~K}$. For example, $\mathrm{Cr}-\mathrm{L} 1_{2}$ indicates the ordered phase wherein the ordered lattice positions are occupied by $\mathrm{Cr}$. (NB: This does not necessarily mean the ordered phase has a lower energy than the RSS.) $T_{s}(X)\left(X=\mathrm{L}_{2}, \mathrm{D} 0_{22}, \mathrm{RSS}\right)$ indicates the

temperature at which phase $X$ is stabilized, i.e., its free energy of formation becomes zero. $T_{c}$ represents a "crossover" temperature between the ordered phase $\mathrm{OP}_{0}$ and RSS. $F_{c}\left(\mathrm{eV} /\right.$ atom) denotes the free energy of formation at $T_{c} . \Delta T_{s}$ represents the temperature range up to $T_{m}$, wherein either one of the ordered phases or the RSS phase is realized as the most stable solid phase (MSSP). All temperatures are listed in Kelvin (K).

\begin{tabular}{|c|c|c|c|c|c|c|c|c|c|}
\hline \multirow[b]{2}{*}{ Composite(\#) } & \multirow[b]{2}{*}{$\mathrm{VEC}_{\mathrm{ave}}$} & \multirow[b]{2}{*}{$\bar{T}_{m}$} & \multirow[b]{2}{*}{$\mathrm{OP}_{0}$} & \multicolumn{3}{|c|}{$T_{s}$} & \multirow[b]{2}{*}{$T_{c}$} & \multirow[b]{2}{*}{$F_{\mathrm{c}}$} & \multirow[b]{2}{*}{$\operatorname{MSSP}\left(\Delta T_{s}\right)$} \\
\hline & & & & $\mathrm{L1}_{2}$ & $\mathrm{D} 0_{22}$ & RSS & & & \\
\hline $\mathrm{CrMnFeCo}(1)$ & 7.50 & 1820 & $\mathrm{Cr}-\mathrm{L} 1_{2}$ & 380 & 683 & 638 & 1016 & -0.045 & $\begin{array}{l}\mathrm{L}_{2}(380 \sim 1016) \\
\operatorname{RSS}\left(1016 \sim \bar{T}_{m}\right)\end{array}$ \\
\hline $\mathrm{CrMnFeNi}(2)$ & 7.75 & 1810 & $\mathrm{Cr}-\mathrm{L}_{2}$ & 448 & 737 & 657 & 963 & -0.037 & $\begin{array}{l}\mathrm{L}_{2}(448 \sim 963) \\
\operatorname{RSS}\left(963 \sim \bar{T}_{m}\right)\end{array}$ \\
\hline CrMnCoNi(3) & 8.00 & 1799 & $\mathrm{Cr}-\mathrm{L} 1_{2}$ & 282 & 553 & 574 & 1003 & -0.051 & $\begin{array}{l}\mathrm{L1}_{2}(282 \sim 1003) \\
\operatorname{RSS}\left(1003 \sim \bar{T}_{m}\right)\end{array}$ \\
\hline $\mathrm{CrMnFeCu}(4)$ & 8.00 & 1717 & $\mathrm{Cr}-\mathrm{L} 1_{2}$ & 2594 & 2755 & 1820 & 687 & 0.135 & unstable \\
\hline CrFeCoNi(5) & 8.25 & 1872 & $\mathrm{Cr}-\mathrm{L} 1_{2}$ & 0 & 127 & 494 & 1333 & -0.100 & $\begin{array}{c}\mathrm{L} 1_{2}(0 \sim 1333) \\
\operatorname{RSS}\left(1333 \sim \bar{T}_{m}\right)\end{array}$ \\
\hline $\mathrm{CrMnCoCu}(6)$ & 8.25 & 1706 & $\mathrm{Cr}-\mathrm{L1}_{2}$ & 2251 & 2425 & 1649 & 768 & 0.105 & $\operatorname{RSS}\left(1649 \sim \bar{T}_{m}\right)$ \\
\hline $\mathrm{CrFeCoCu}(7)$ & 8.50 & 1779 & $\mathrm{Cr}-\mathrm{L} 1_{2}$ & 1913 & 1917 & 1469 & 819 & 0.078 & $\operatorname{RSS}\left(1469 \sim \bar{T}_{m}\right)$ \\
\hline $\mathrm{CrMnNiCu}(8)$ & 8.50 & 1696 & $\mathrm{Cr}-\mathrm{L}_{2}$ & 1881 & 1950 & 1398 & 691 & 0.085 & $\operatorname{RSS}\left(1398 \sim \bar{T}_{m}\right)$ \\
\hline $\mathrm{MnFeCoNi}(9)$ & 8.50 & 1706 & $\mathrm{Mn}-\mathrm{L}_{2}$ & 0 & 128 & 333 & 970 & -0.076 & $\begin{array}{c}\mathrm{L1}_{2}(0 \sim 970) \\
\operatorname{RSS}\left(970 \sim \bar{T}_{m}\right)\end{array}$ \\
\hline $\mathrm{CrFeNiCu}(10)$ & 8.75 & 1769 & $\mathrm{Cr}-\mathrm{DO}_{22}$ & 1786 & 1546 & 1223 & 750 & 0.074 & $\operatorname{RSS}\left(1223 \sim \bar{T}_{m}\right)$ \\
\hline $\mathrm{MnFeCoCu}(11)$ & 8.75 & 1614 & $\mathrm{Mn}-\mathrm{L} 1_{2}$ & 1597 & 1665 & 1218 & 663 & 0.066 & $\operatorname{RSS}\left(1218 \sim \bar{T}_{m}\right)$ \\
\hline $\mathrm{CrCoNiCu}(12)$ & 9.00 & 1758 & $\mathrm{Cr}-\mathrm{DO}_{22}$ & 1711 & 1594 & 1117 & 417 & 0.092 & $\operatorname{RSS}\left(1117 \sim \bar{T}_{m}\right)$ \\
\hline $\mathrm{MnFeNiCu}(13)$ & 9.00 & 1604 & $\mathrm{Mn}-\mathrm{DO}_{22}$ & 1395 & 1205 & 956 & 592 & 0.057 & $\operatorname{RSS}\left(956 \sim \bar{T}_{m}\right)$ \\
\hline $\mathrm{MnCoNiCu}(14)$ & 9.25 & 1593 & $\mathrm{Mn}-\mathrm{DO}_{22}$ & 1497 & 1204 & 981 & 653 & 0.060 & $\operatorname{RSS}\left(981 \sim \bar{T}_{m}\right)$ \\
\hline $\mathrm{FeCoNiCu}(15)$ & 9.50 & 1666 & $\mathrm{Fe}-\mathrm{L}_{2}$ & 421 & 474 & 469 & 539 & -0.008 & $\begin{array}{l}\mathrm{L1}_{2}(421 \sim 539) \\
\operatorname{RSS}\left(539 \sim \bar{T}_{m}\right)\end{array}$ \\
\hline
\end{tabular}

for large VEC values ( $\geq 8.50)$, except for $\mathrm{MnFeCoCu}(11)$ and $\mathrm{FeCoNiCu}(15)$. However, $\mathrm{D}_{22}$ ordered phases do not appear as the most stable structure, because there is no condition that the free energy of formation is negative and smaller than the RSS in the entire temperature range. In contrast, alloys with large VEC values and $\mathrm{Cu}$ as a constituent exhibit the RSS phases. Finally, we may conclude that neither entropy nor enthalpy is dominant in stabilizing HEAs, but both cooperatively stabilize their structures (see Figure 3 ) depending on temperature and other factors such as VEC.

\section{B. Energetics}

A clear understanding of the energetics of each phase is important to determine the free energy of formation at a finite temperature for discussing the phase stability of HEAs. Figure 4 shows the average free energies of formation of 100 different configurations, with the standard deviation at $1000 \mathrm{~K}$ for all 15 quaternary alloys, each of which has eight types of ordered phases and the RSS phase. Ordered phases with the lowest energy at $0 \mathrm{~K}\left(\mathrm{OP}_{0}\right)$ for each quaternary alloy are listed in Table I] For all quaternary alloys (Fig. S-1), the free energy of formation of at least one ordered phase is lower than that of the RSS phase at $0 \mathrm{~K}$. Interestingly, the $\mathrm{D}_{22}$ ordered phases exhibit a lower free energy of formation than the $\mathrm{L}_{2}$ ordered phases in the high VEC region in some alloys. However, because the free energy of formation of $\mathrm{D}_{22}$ ordered phases is not negative below the RSS value, the $\mathrm{D}_{22}$ phases are not stable phase in the entire temperature range.

As shown in Fig. 4. $\mathrm{CrFeNiCu}\left(10 ; \mathrm{VEC}_{\mathrm{ave}}=8.75\right), \mathrm{Cr}-$ $\mathrm{CoNiCu}\left(12 ; \mathrm{VEC}_{\mathrm{ave}}=9.0\right), \mathrm{MnFeNiCu}\left(13 ; \mathrm{VEC}_{\mathrm{ave}}=9.0\right)$, and $\mathrm{MnCoNiCu}\left(14 ; \mathrm{VEC}_{\mathrm{ave}}=9.25\right)$ exhibit $\mathrm{D}_{22}$ phases with lower energies than that of the $\mathrm{L}_{2}$ phases. These alloys demonstrate that the free energy of $\mathrm{L}_{2}$ increases remarkably, as a result, these free energies of formation show a positive value at $1000 \mathrm{~K}$. This result means that these ordered structures are thermodynamically unstable, however, we compare the free energies of $\mathrm{L}_{2}$ and $\mathrm{D}_{22}$ to get insight of a competitive cooperation. Similar competitive behavior between the phase stability of $\mathrm{L}_{2}$ and $\mathrm{D}_{22}$ for a wide range of VECs was previously reported for $\mathrm{Ni}_{3} \mathrm{~V}, \mathrm{Pd}_{3} \mathrm{~V}$, and $\mathrm{Pt}_{3} \mathrm{~V}$, which are fundamental binary alloys, in contrast with HEAs [34]. The VEC at which the crossover of the energy difference between the $\mathrm{L}_{2}$ and $\mathrm{D}_{22}$ phases occurs in this study $\left(\mathrm{VEC}_{\mathrm{ave}}=8.5\right)$ is comparable to the value reported for the previously stud- 


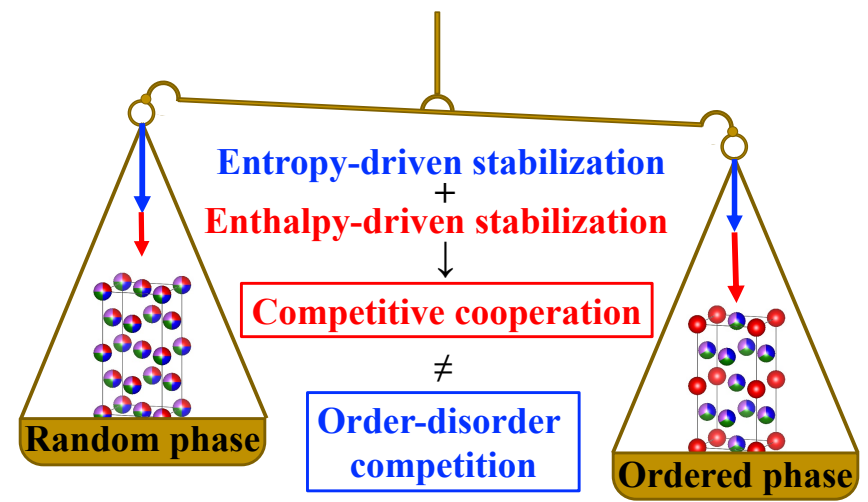

FIG. 3: Phase stability of 3d-transition metal high-entropy alloys (HEAs) is realized by an order-disorder "competitive cooperation," rather than a usual order-disorder competition. The former was newly proposed in this study, while the latter has already been accepted in the HEA community. [25]

ied binary system [34]. One key result obtained from Fig. 4 is that the systems comprising $\mathrm{Cu}$ have high free energies of formation. Moreover, quaternary alloys in which $\mathrm{Cu}$ forms the ordered phase entail higher free energies of formation of the ordered phase than those of the RSS phase. We attribute the high energies of formation associated with $\mathrm{Cu}$ to the positive formation energies exhibited by the least energetic structures of the binary compounds consisting of $\mathrm{Cu}$ and $3 \mathrm{~d}$ transition metals (Cr, Mn, Fe, Co, and Ni) [63], [64], [65]. The present results are consistent with previous studies because as the number of bonding pairs between $\mathrm{Cu}$ and other $3 \mathrm{~d}$ transition metals increased in the $\mathrm{Cu}$-ordered phases (cf. Table III).

\section{Magnetic ordering}

Figure 5 illustrates the distribution of magnetic moments as a function of the average magnetic moment at the first nearestneighbor sites in the most energetically stable ordered phase for 15 quaternary alloys. In Fig. 5, we observe certain common trends: "Cr" and "Mn without Cr" show antiferromagnetic properties, "Mn with $\mathrm{Cr}$ ", "Fe", and "Co" show ferromagnetic properties, and "Mn with a ferromagnetic moment" shows values that can be compared to those of Fe. In contrast, Mn with antiferromagnetic properties behave similar to $\mathrm{Cr}$. Therefore, the magnetic nature of $\mathrm{Mn}$ depends on the presence or absence of $\mathrm{Cr}$. $\mathrm{Ni}$ and $\mathrm{Cu}$ exhibit nonmagnetic (paramagnetic) behavior in all systems. Although the magnetic moments are mostly similar, the magnetic moment distributions of some alloys show that the average magnetic moments at the first nearest-neighbor sites are discrete; for example, at $\mathrm{Cr}$ and $\mathrm{Fe}$ in $\mathrm{CrFeNiCu}\left(\mathrm{VEC}_{\text {ave }}=8.75\right)$, as shown in Fig. 4(10). Such a discrete distribution can be attributed to the number of ferromagnetic or antiferromagnetic atoms located at the first nearest-neighbor sites of the FCC lattice (Fig. 2(b) in [25]). For example, the antiferromagnetic atoms in the or- dered phases are surrounded by four ferromagnetic atoms on average because the other three elements are occupied at the 12 first nearest-neighbor sites.

Niu et al. [25] previously evaluated the magnetic moment of each element and the average magnetic moment of the 12 first-nearest neighbor sites for the RSS and $\mathrm{Cr}-\mathrm{L} 1_{2}$ ordered phases of the CrFeCoNi quaternary alloy $\left(\mathrm{VEC}_{\mathrm{ave}}=8.25\right)$, and they are the same as that of the composite shown in Fig. 4 (5). The results obtained in this study demonstrate that Fe and Co exhibit ferromagnetic properties, whereas $\mathrm{Cr}$ exhibits anti-ferromagnetic properties. Since the results obtained are consistent with those reported previously [25], the validity of the calculation methods described herein can be confirmed. Moreover, Niu et al. reported a discrete distribution of the average magnetic moments at the first nearest-neighbor sites in the $\mathrm{Cr}-\mathrm{Ll}_{2}$ ordered phase. They revealed that the magnetism of $\mathrm{Cr}$ in the $\mathrm{CrFeCoNi}$ quaternary alloy is strongly influenced by the magnetism of the first nearest-neighbor sites [25]. Zuo et al. [66] evaluated the magnetic moments of the individual atoms of the FCC MnFeCoNi quaternary alloy and FCC CrM$\mathrm{nFeCoNi}$ quinary alloy and reported that $\mathrm{Fe}$ exhibits a higher magnetic moment than $\mathrm{Co}, \mathrm{Ni}$ exhibits a near-zero magnetic moment, and $\mathrm{Cr}$ exhibits antiferromagnetism. In addition, $\mathrm{Mn}$ atoms cause a wider distribution in the magnetic moments of atoms and exhibit both ferromagnetism and antiferromagnetism in the RSS phase. [66]

Ghazisaeidi et al. reported a similar magnetism distribution in the hexagonal close packed (HCP) and FCC phases of CrCoNi, FeCoNi, MnFeNi, MnCoNi, and CrMnFeCoNi. [44] The magnetism of $\mathrm{Cr}$ and $\mathrm{Mn}$ atoms ranges from ferromagnetism to antiferromagnetism in the RSS phase. Fedorov et al. [67] and Schönfeld et al. [68] out that the magnetic properties of the constituent elements are key factors governing phase stability and the formation of ordered phases in $3 \mathrm{~d}$ transition-metal-based alloys.

\section{Quantitative Structure-Property Relationship}

The $\mathrm{L}_{2}$ and $\mathrm{D}_{22}$ phases have typical ordered crystal structures in the 25:75 (at.\%) FCC binary alloys. For example, the $\mathrm{L}_{2}$ structure can be observed in many alloys with a 3:1 composition, such as $\mathrm{Cu}_{3} \mathrm{Au}, \mathrm{Ni}_{3} \mathrm{Al}$, and $\mathrm{TiPt}_{3}$; similarly, the $\mathrm{DO}_{22}$ structure can be observed in trialuminides $\mathrm{Al}_{3}(\mathrm{Sc}, \mathrm{Ti}, \mathrm{V})$ and $\mathrm{Ni}_{3} \mathrm{~V}$. Although the appearance of the $\mathrm{L}_{2}$ and $\mathrm{DO}_{22}$ phases in FCC binary alloys has been discussed previously, only a few studies have focused on the configuration of the neighboring sites in multiprincipal-element alloys. [25] The number of first, second, third, and fourth nearest-neighbor sites in the $\mathrm{L}_{2}$ and $\mathrm{DO}_{22}$ phases are $12,6,24$, and 12 , respectively (Table II). The 25 at.\% element in the $\mathrm{L}_{2}$ and $\mathrm{D}_{22}$ phases is not located at the first nearest neighbor sites, and thus, they can be isolated from each other and surrounded by the 75 at.\% elements. For instance, $\mathrm{Au}$ atoms in the $\mathrm{L}_{2}$ phase of $\mathrm{Cu}_{3} \mathrm{Au}$ are not located at their first nearest-neighbor sites and are surrounded by $12 \mathrm{Cu}$ atoms.

Figure 6 shows the relationship between the formation energy and occupancy rate of $\mathrm{Cr}-\mathrm{Cr}$ pairs at the first and sec- 


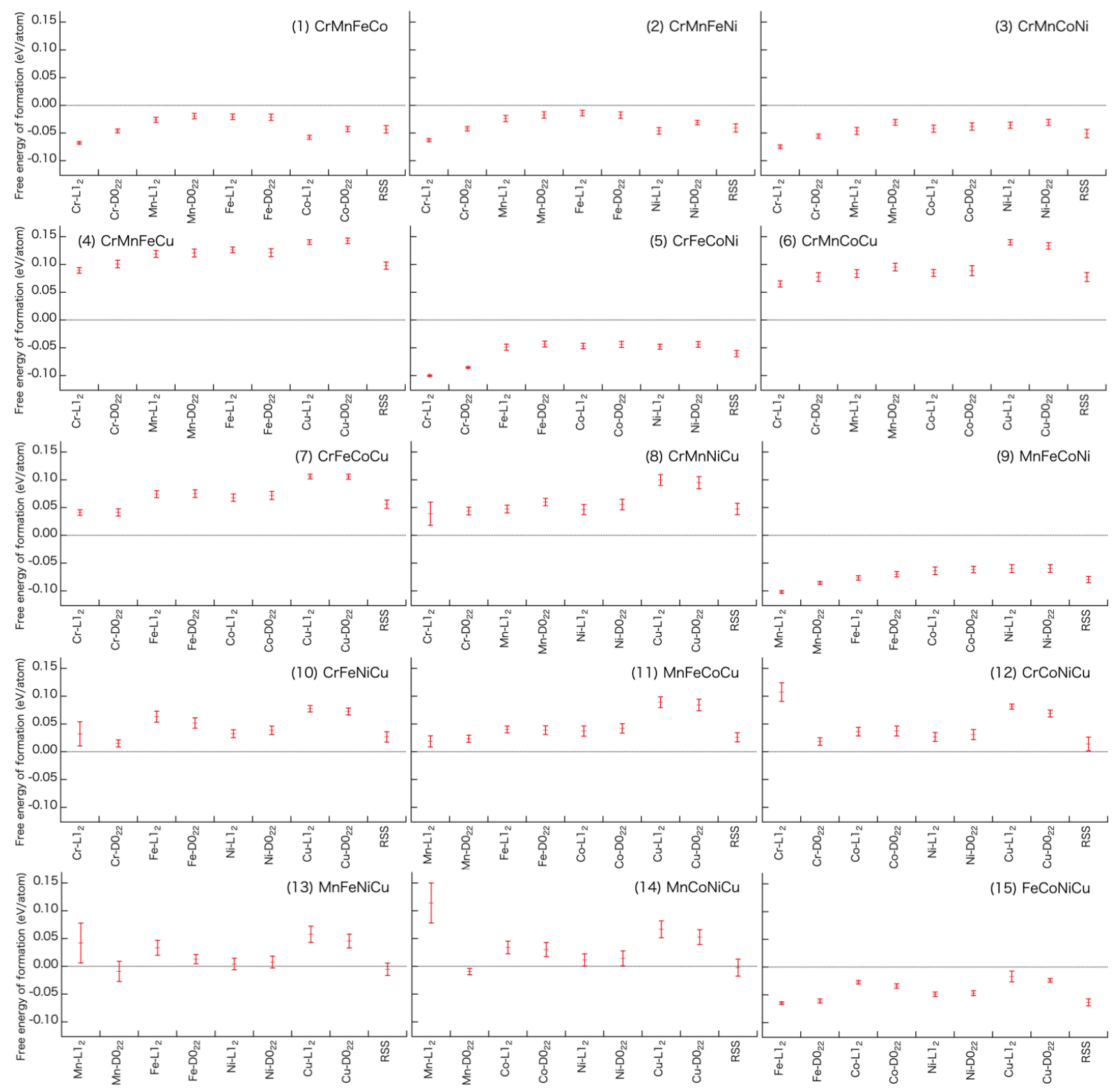

FIG. 4: Free energy of formation at $1000 \mathrm{~K}$ for 15 cases of quaternary alloys listed in Table $\mathrm{I}$. Error bars indicate the standard deviations (SD) for each phase. The original raw data for each phase are shown in Fig. S-2 (Supplementary Information).

ond nearest-neighbor sites in the RSS phase of the $\mathrm{CrFeCoNi}$ quaternary alloy. In the RSS phase, the rate of occupancy by the same element can remain at 25 at.\% on average at any nearest-neighbor site. Meanwhile, the occupancy rates for the ordered phases depend on the nearest-neighbor site, as listed in TABLE II] For the first nearest-neighbor sites (Fig. 6(a)), the calculated results reveal a lowering of the energy with a decreasing occupancy rate, such that the energy approaches the values corresponding to the $\mathrm{L}_{2}$ and $\mathrm{D}_{22}$ ordered phases on extrapolation of the occupancy rate to zero. However, for the second nearest-neighbor sites (Fig.6(b)), an opposite trend is observed, i.e., the formation energies decrease with increasing occupancy rate. The values for both ordered phases can be found on the extrapolation line. These results imply that the configurations at the first and second nearest-neighbor sites affect the formation energy of the $\mathrm{CrFeCoNi}$ quaternary alloy. Furthermore, the lower formation energies of the ordered phases can be attributed to their unique occupancy rates. 


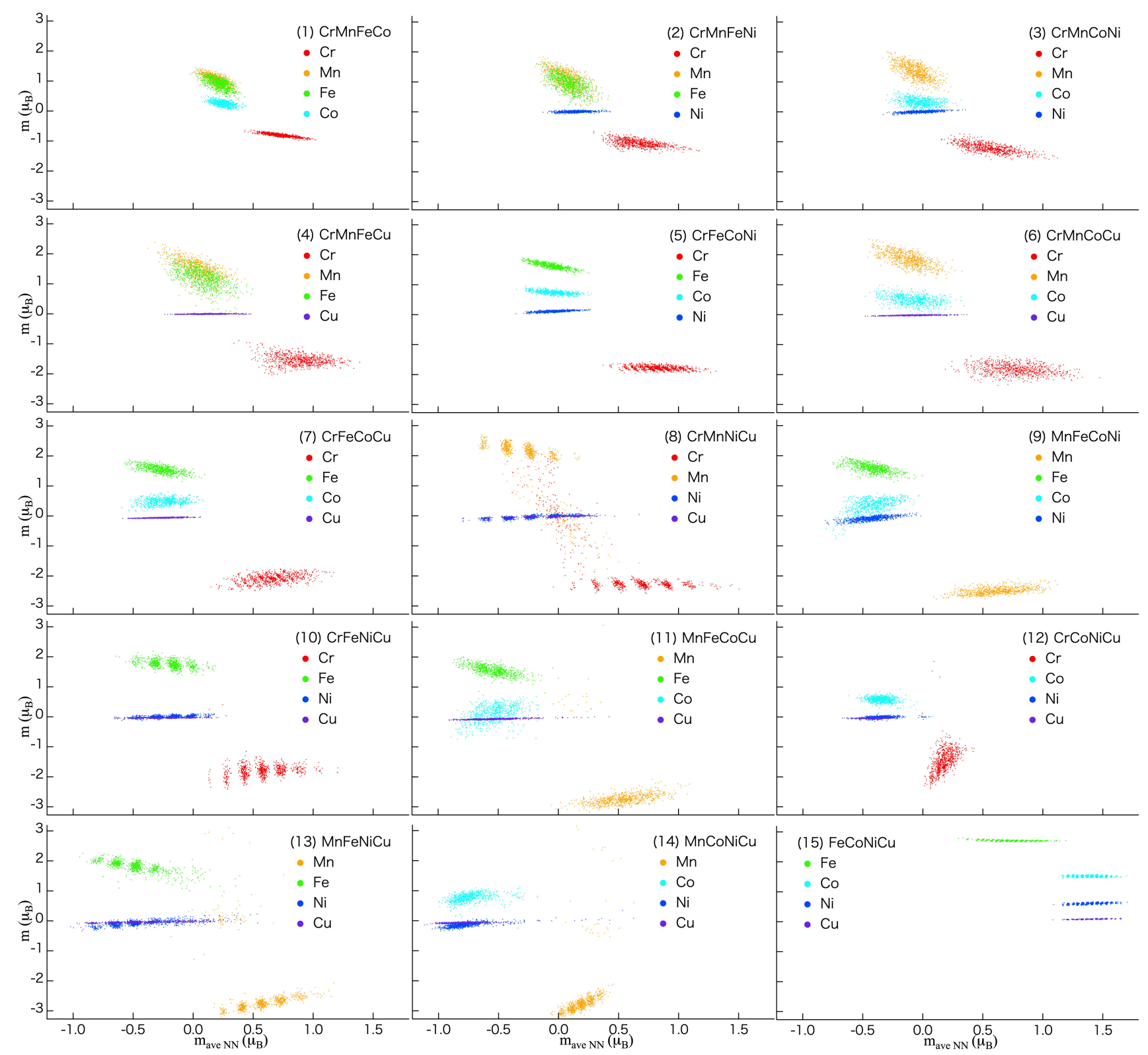

FIG. 5: Distribution of magnetic moments as a function of the average magnetic moment at the first nearest-neighbor site in the most energetically stable ordered phase for the 15 quaternary alloys listed in Table $\mathbb{T}$ as $\mathrm{OP}_{0}$. Red, orange, green, light blue, dark blue, and purple indicate $\mathrm{Cr}, \mathrm{Mn}, \mathrm{Fe}, \mathrm{Co}, \mathrm{Ni}$, and $\mathrm{Cu}$, respectively. $m$ and $m_{\text {aveNN }}$ represent the magnetic moment for each atom and the average magnetic moment of the 12 first nearest-neighbor sites, respectively.

Figures 7 (a) and (b) show the bond-length distributions in the RSS and $\mathrm{Cr}-\mathrm{Ll}_{2}$ ordered phases, respectively. In Figure 7 , the solid lines indicate homogeneous element pairs, whereas the dotted and dashed lines indicate heterogeneous element pairs. Many $3 \mathrm{~d}$ transition metals, especially $\mathrm{Cr}, \mathrm{Fe}, \mathrm{Co}$, and $\mathrm{Ni}$, exhibit comparable atomic radii. However, the $\mathrm{CrFeCoNi}$ quaternary alloy shows a different bond-length distribution. These results indicate that these element pairs have different interatomic distances. As shown in Figure 7(a), the $\mathrm{Cr}-\mathrm{Cr}$ pairs in the RSS phase exhibit a broad bond-length distribution and longer bond lengths than those corresponding to the first nearest-neighbor sites, as estimated by the lattice constant. The bond lengths of the homogeneous element pairs are in a decreasing order of $\mathrm{Cr}, \mathrm{Fe}, \mathrm{Ni}$, and $\mathrm{Co}$. The same trend is observed for the $\mathrm{Cr}-\mathrm{L}_{2}$ ordered phase in Fig. 77b). Notably, there are no $\mathrm{Cr}-\mathrm{Cr}$ pairs in the $\mathrm{Cr}-\mathrm{Ll}_{2}$ ordered phase because $\mathrm{Cr}$ atoms occupy the sites constructing the framework of the $\mathrm{L1}_{2}$ ordered phase, and they are not located at the first nearest- 
TABLE II: Comparison of the site occupancies of $\mathrm{L}_{2}, \mathrm{D}_{22}$, and RSS phases containing 25 at.\% of ordered elements. For each neighbor site therein, the number of the ordered elements over the total number of the sites is given with its percentage in brackets. For the RSS phase, the occupancy rate for any site is 25 at.\% on average (shown in brackets).

\begin{tabular}{crrr} 
Neighbor site & \multicolumn{1}{c}{$\mathrm{L}_{2}$} & \multicolumn{1}{c}{$\mathrm{D}_{22}$} & $\mathrm{RSS}$ \\
\hline First nearest & $0 / 12\langle 0\rangle$ & $0 / 12\langle 0\rangle$ & $\langle 25\rangle$ \\
Second nearest & $6 / 6\langle 100\rangle$ & $4 / 6\langle 67\rangle$ & $\langle 25\rangle$ \\
Third nearest & $0 / 24\langle 0\rangle$ & $8 / 24\langle 33\rangle$ & $\langle 25\rangle$ \\
Fourth nearest & $12 / 12\langle 100\rangle$ & $4 / 12\langle 33\rangle$ & $\langle 25\rangle$ \\
\hline
\end{tabular}
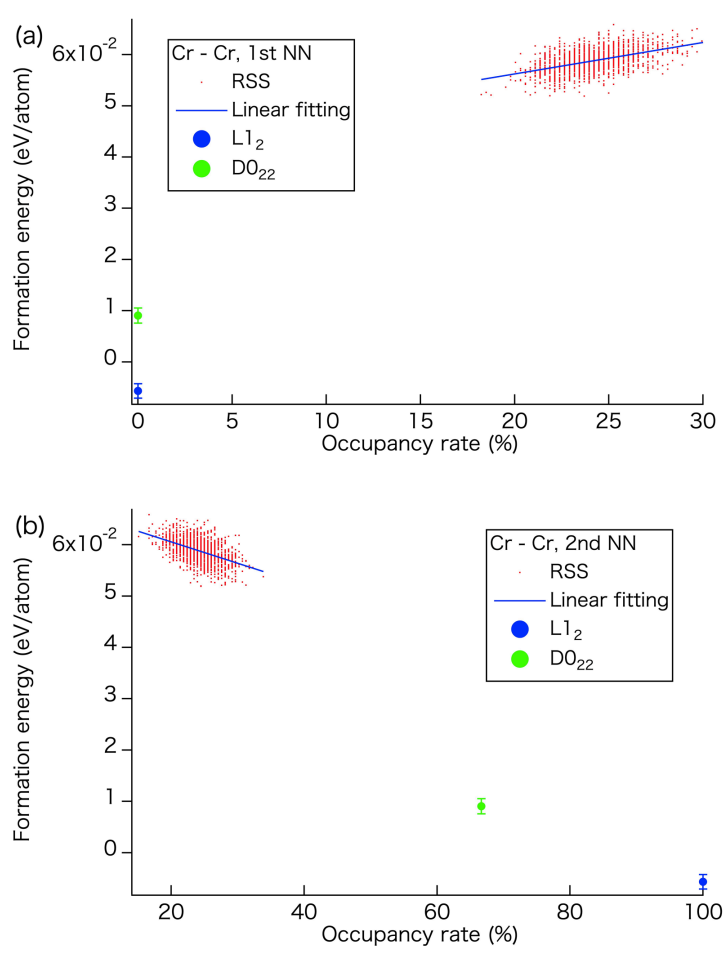

FIG. 6: Dependence of the formation energies of ordered and RSS phases in the $\mathrm{CrFeCoNi}$ quaternary alloy on the occupancy rate of the $\mathrm{Cr}-\mathrm{Cr}$ pairs at the (a) first and (b) second nearest-neighbor sites. The red dots represent the RSS phase obtained using 1,500 samples, whereas the blue lines serve as guidelines. The blue and green dots indicate the $\mathrm{Cr}-\mathrm{L} 1_{2}$ and $\mathrm{Cr}-\mathrm{D} 0_{22}$ ordered phases, respectively.

neighbor sites (TABLE [I]). Although the structure optimization process is applied to all atoms in the $\mathrm{Cr}-\mathrm{Ll}_{2}$ ordered and RSS phases, the bond-length distributions of the $\mathrm{Cr}-\mathrm{L} 1_{2}$ ordered phase are narrower than those of the RSS phase.

Let us consider the half widths at half maximum (HWHM) of the fitted Gaussian functions. The HWHMs of Fe-Fe, $\mathrm{Co}-\mathrm{Co}$, and $\mathrm{Ni}-\mathrm{Ni}$ in the $\mathrm{L}_{2}$ ordered phases are $64.5 \%$, $71.2 \%$, and $68.6 \%$, respectively, of the corresponding RSS phases. It is presumed that the Cr occupancy at the second
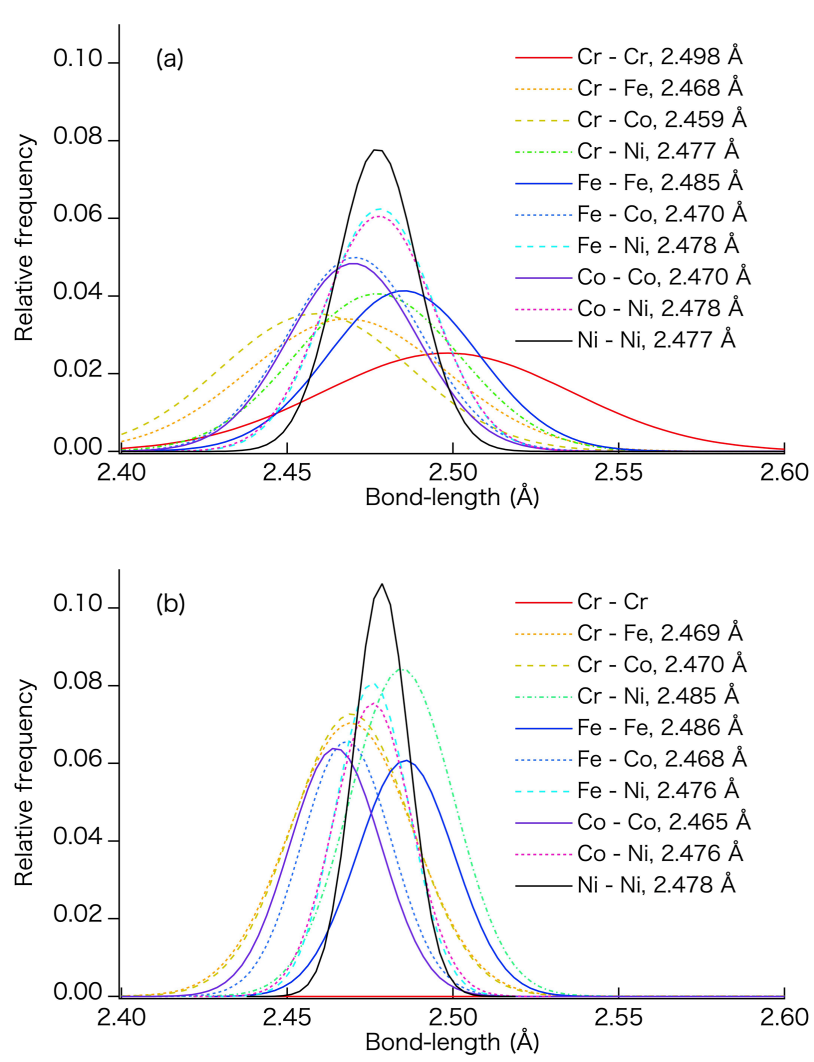

FIG. 7: Relative frequency distribution of bond-lengths fitted with Gaussian function for each element pair in the (a) RSS phase and (b) $\mathrm{Cr}-\mathrm{L} 1_{2}$ ordered phase of the $\mathrm{CrFeCoNi}$ quaternary alloy after structure optimization. The bond lengths in the legends indicate the average values obtained upon fitting with the Gaussian function.

nearest-neighbor sites results in a small bond-length distribution with regularity. Gao and Alman used ab initio molecular dynamics (AIMD) simulations [69] and reported that the $\mathrm{Al}_{1.3} \mathrm{CrFeCoNiCu}$ senary alloy exhibits a different distribution for each element pair, which is similar to the results obtained in this study for 3d-transition-metal-based equiatomic quaternary alloys. However, the HfNbTaTiZr quinary alloys show almost the same distribution for all its constituent elements without a 3d transition metal. [69] In this study, we conclude that the bond-length distributions of the described systems are significantly different because each $3 \mathrm{~d}$ transition metal has an individual magnetic behavior.

\section{SUMMARY AND FUTURE PROSPECTS}

In this study, we performed first-principles calculations considering the configurational entropy term to investigate the effect of VEC and temperature on the stabilities of the two ordered phases: $\mathrm{L}_{2}$ and $\mathrm{D}_{22}$. Further, we drew a comparison with the disordered atomic configurations in equiatomic qua- 
ternary alloys comprising $\mathrm{Cr}, \mathrm{Mn}, \mathrm{Fe}, \mathrm{Co}, \mathrm{Ni}$, and $\mathrm{Cu}$. We found that the VEC is a significant factor controlling phase stability in both ordered phases. In the high-VEC region, the $\mathrm{D}_{22}$ ordered phase is energetically more stable than the $\mathrm{L}_{2}$ ordered phase, though both phases are metastable. The magnetic moments indicate that the anti-ferromagnetic $\mathrm{Cr}$ and $\mathrm{Mn}$ atoms are surrounded by the ferromagnetic $\mathrm{Fe}, \mathrm{Co}$, and $\mathrm{Ni}$ atoms located at the first nearest-neighbor sites. Such magnetic configurations make the ordered HEA phases energetically stable. Since the constituent elements corresponding to 25 at. $\%$ in the $\mathrm{L}_{2}$ and $\mathrm{DO}_{22}$ ordered phases exhibit no bonding between the first nearest-neighbor sites, the extent of the combination of anti-ferromagnetic and ferromagnetic elements can be increased in the $\mathrm{L}_{2}$ and $\mathrm{DO}_{22}$ phases. The bondlength distribution results reveal that the $\mathrm{Cr}-\mathrm{Cr}$ distances are longer and the distributions are broader than those of other element pairs, which suggests that the high formation energy of the RSS phase can be attributed to the abnormal bond length between the anti-ferromagnetic atoms. Consequently, both the ordered phases have enthalpy-driven energetically favorable structures that are enthalpy-driven.

Since 3d-transition-metal quaternary systems are base alloys for their extension to more multicomponent systems, a comprehensive understanding of their structures would serve as a stepping stone toward efficient HEA design. As shown for most 3d-transition-metal quaternary alloys, we should consider that phase stability is realized by an order-disorder "competitive cooperation," rather than a usual order-disorder competition. The former was newly proposed in this study, while the latter has already been accepted in the HEA community. Most researchers in the HEA community naively consider that the "fully disordered/random atomic configurations" of HEAs have higher entropies, and therefore, they have higher phase stabilities. However, it is important to recognize the cooperation between "a magnetically ordered atomic configuration composed of one element" and "fully disordered/random configurations composed of the other three elements," which correspond to the enthalpy and entropy terms, respectively, to understand the microscopic structures of HEAs in detail.

In this study, the enthalpy terms that describe structural properties were evaluated by first-principles calculations to obtain insight into an atomistic origin of the energetics of the 3d-transition-element HEAs. Since the entropy term is as important as the enthalpy term, the addition of a configurational entropy term in the density functional theory (DFT) calculations is effective for determining the crystal structure of HEAs. This enables us to estimate the transition temperatures between the ordered and RSS phases, which is useful for obtaining the relationship between structure and processing. It is already widely recognized in the materials science field that the relationship among structures, properties, and processing methods is a crucial factor in realizing outstanding structural materials [70]. For example, Kenel et al. designed a 3d-transition-metal HEA using a 3D printing technique [71] with oxide nanopowder, which exhibits prominent mechanical properties at ambient and cryogenic temperatures [72]. Futhermore, it is necessary to predict an accurate phase di- agram of HEA to realize QSPR by combining the calculation of the phase diagram (CALPHAD) [73] and the firstprinciples based thermodynamic assessment [74]. We believe that the systematic investigation described herein will be helpful for realizing such an assessment and for predicting the microstructures of HEA by performing model simulations such as "first-principles phase field model" without any thermodynamic empirical parameters [75].

Finally, we note that our finding is applicable to "ceramics", "oxides," and "carbide", as well because the "high-entropy" concept has been adopted even in them [76-83]. Furthermore, our proposed concept sheds light on the constituent phases of even multiprincipal element alloys (MPEAs) [84, 85] and multiphase compositionally complex alloys (CCAs) [86]. Thus, our finding can encourage experimentalists to investigate the microscopic identification of their structures, which will help us elucidate their QSPR.

\section{SUPPLEMENTARY INFORMATION}

\section{A. Ab initio calculations}

Figure S-1 shows the formation free energy as a function of temperature for 15 kinds of quaternary alloys, which are listed at Table I. The slope of the formation free energy for RSS phase is steeper than that of the ordered phases, because the entropy term of RSS is larger than that of the ordered phases. On the other hand, the slope of both ordered phases are exact same. Consequently, the comparison of the formation free energy of the ordered phases is determined by formation energy at $0 \mathrm{~K}$. Note that in the case of $\mathrm{CrFeCoCu}\left(\mathrm{VEC}_{\mathrm{ave}}=8.50\right)$ alloy in (7) at Fig. S-1, the both ordered phases have almost same formation free energy. Figure S-1 (14) and Figure 2 show the formation free energy for $\mathrm{MnCoNiCu}$ alloy with different vertical scale. In Fig. S-1, different scale is set for comparison with other alloy systems.

Figure S-2 illustrates the distribution of the formation free energies of at $1,000 \mathrm{~K}$ for quaternary alloys using $100 \mathrm{sam}$ ples. Figure 3 shows the average of formation free energies with the standard deviation from the raw data of 100 samples in Fig. S-2. $X-\mathrm{Ll}_{2}$ and $X-\mathrm{D} 0_{22}(X=\mathrm{Cr}, \mathrm{Mn}, \mathrm{Fe}, \mathrm{Co}, \mathrm{Ni}$, and $\mathrm{Cu}$ ) indicate the cubic corner (CC) sites of an face centered cubic (FCC) lattice occupied by each element for $\mathrm{L}_{2}$ structure and the corner sites and body centered sites of an FCC lattice when $c=2 a$ for $\mathrm{D}_{22}$ structure, respectively. a is a lattice constant of FCC lattice. RSS is random solid solution (cf. Figure 1). Namely, 8 kinds of the ordered phases and RSS phase for each composite can be compared in Fig. S-1. It becomes clear that the quaternary alloys containing $\mathrm{Cu}$ show high formation free energy than those of other alloys, as a result of comparison of 15 kinds of the alloys with the same energy ranges on all vertical axes. A few ordered phases, such as $\mathrm{Cr}-\mathrm{Ll}_{2}$ phase, show a narrow dispersion of formation free energy, while many ordered phases show a same wide dispersion as RSS phases. Interestingly, $X-\mathrm{L1}_{2}(X=\mathrm{Cr}$ and $\mathrm{Mn})$ phases of $\mathrm{CrMnNiCu}\left(\mathrm{VEC}_{\mathrm{ave}}=8.50\right)$ in $(8), \mathrm{CrFeNiCu}\left(\mathrm{VEC}_{\mathrm{ave}}=\right.$ $8.75)$ in (10), $\mathrm{MnFeCoCu}\left(\mathrm{VEC}_{\mathrm{ave}}=8.75\right)$ in $(11), \mathrm{CrCoNiCu}$ 
$\left(\mathrm{VEC}_{\mathrm{ave}}=9.0\right)$ in (12), $\mathrm{MnFeNiCu}\left(\mathrm{VEC}_{\mathrm{ave}}=9.0\right)$ in (13), and $\mathrm{MnCoNiCu}\left(\mathrm{VEC}_{\mathrm{ave}}=9.25\right)$ in (14) at Fig. S-2 show a scatter distribution.

\section{Acknowledgements}

This study was supported by the computational resources of the HPCI system (Project ID: hp190014, hp190059, hp200040, hp210019), the JHPCN system (Project ID: jh190019), Institute for Materials Research, Tohoku University (Proposal No. 19S0501, 20S0505, 202012-SCKXX0506), JAIST, and NIMS. H.M. thanks the Korea Institute of
Science and Technology (Grant Nos. 2Z05840, No. 2E31201, No. 2N62110) for providing the financial support for this research. R.S. acknowledges the support from the Council for Science, Technology and Innovation under the Crossministerial Strategic Innovation Promotion Program. K.H. acknowledges the financial support from MEXT-KAKENHI (Grant Nos. JP19K05029, JP19H05169) and the Air Force Office of Scientific Research (Award Number: FA2386-20-14036).
[1] M. C. Gao, J.-W. Yeh, P. Liaw, Y. Zhang, High-entropy alloys: Fundamentals and applications, Springer, 2016. doi: 10. $1007 / 978-3-319-27013-5$

[2] E. George, W. Curtin, C. Tasan, High entropy alloys: A focused review of mechanical properties and deformation mechanisms, Acta Materialia 188 (2020) 435-474. doi: https://doi.org/10.1016/j.actamat.2019.12.015 URL https://www.sciencedirect.com/science/ article/pii/S1359645419308444

[3] C. Varvenne, A. Luque, W. A. Curtin, Theory of strengthening in fcc high entropy alloys, Acta Materialia 118 (2016) 164176. doi:https://doi.org/10.1016/j.actamat. 2016 . 07.040

URL https://wWw.sciencedirect.com/science/ article/pii/S1359645416305481

[4] C. Varvenne, G. Leyson, M. Ghazisaeidi, W. Curtin, Solute strengthening in random alloys Acta Materialia 124 (2017) 660-683. doi:https://doi.org/10.1016/j.actamat. 2016.09.046

URL https://www.sciencedirect.com/science/ article/pii/S1359645416307455

[5] D. Wei, X. Li, S. Schönecker, J. Jiang, W.-M. Choi, B.-J. Lee, H. S. Kim, A. Chiba, H. Kato, Development of strong and ductile metastable face-centered cubic single-phase high-entropy alloys, Acta Materialia 181 (2019) 318-330. doi:https: //doi.org/10.1016/j.actamat.2019.09.050 URL https://www. sciencedirect.com/science/ article/pii/S1359645419306408

[6] F. Maresca, W. A. Curtin, Mechanistic origin of high strength in refractory bcc high entropy alloys up to 1900k Acta Materialia 182 (2020) 235-249. doi:https: //doi.org/10.1016/j.actamat.2019.10.015 URL https://wWw.sciencedirect.com/science/ article/pii/S1359645419306755

[7] L. Han, Z. Rao, I. R. Souza Filho, F. Maccari, Y. Wei, G. Wu, A. Ahmadian, X. Zhou, O. Gutfleisch, D. Ponge, D. Raabe, Z. Li, Ultrastrong and ductile soft magnetic high-entropy alloys via coherent ordered nanoprecipitates. Advanced Materials 33 (37) (2021) 2102139. arXiv:https://onlinelibrary. wiley.com/doi/pdf/10.1002/adma.202102139. doi:https://doi.org/10.1002/adma.202102139 URL https://onlinelibrary.wiley.com/doi/abs/10. 1002/adma. 202102139

[8] Y. Sun, S. Dai, High-entropy materials for catalysis: A new frontier Science Advances 7 (20) (2021).

URL https://advances.sciencemag.org/content/7/ 20/eabg1600

[9] Y. Mizuguchi, M. R. Kasem, T. D. Matsuda, Supercon- ductivity in cual2-type co0.2ni0.1cu0.1rh0.3ir0.3zr2 with a high-entropy-alloy transition metal site Materials Research Letters 9 (3) (2021) 141-147.

URL https://doi.org/10.1080/21663831.2020. 1860147

[10] O. El-Atwani, N. Li, M. Li, A. Devaraj, J. K. S. Baldwin, M. M. Schneider, D. Sobieraj, J. S. Wróbel, D. Nguyen-Manh, S. A. Maloy, E. Martinez, Outstanding radiation resistance of tungsten-based high-entropy alloys, Science Advances 5 (3) (2019).

[11] R. Kozak, A. Sologubenko, W. Steurer, Single-phase highentropy alloys - an overview, Zeitschrift für Kristallographie - Crystalline Materials 230 (1) (2015) 55-68.

URL https://doi.org/10.1515/zkri-2014-1739

[12] Y. Ma, Q. Wang, X. Zhou, J. Hao, B. Gault, Q. Zhang, C. Dong, T. G. Nieh, A novel soft-magnetic b2-based multiprincipalelement alloy with a uniform distribution of coherent bodycentered-cubic nanoprecipitates Advanced Materials 33 (14) (2021) 2006723. arXiv:https://onlinelibrary.wiley. com/doi/pdf/10.1002/adma.202006723, doi:https:// doi.org/10.1002/adma.202006723.

URL https://onlinelibrary.wiley.com/doi/abs/10. 1002 /adma. 202006723

[13] N. Savage, Striking a balance with high-entropy alloys Nature 595 (7865) (2021) S4-S5.

URL https://media.nature.com/original/ magazine-assets/d41586-021-01788-0/ d41586-021-01788-0.pdf

[14] Y. Yao, Z. Huang, P. Xie, S. D. Lacey, R. J. Jacob, H. Xie, F. Chen, A. Nie, T. Pu, M. Rehwoldt, D. Yu, M. R. Zachariah, C. Wang, R. Shahbazian-Yassar, J. Li, L. Hu, Carbothermal shock synthesis of high-entropy-alloy nanoparticles, Science 359 (6383) (2018) 1489-1494.

[15] Y. Lederer, C. Toher, K. S. Vecchio, S. Curtarolo, The search for high entropy alloys: A high-throughput ab-initio approach. Acta Materialia 159 (2018) 364-383. doi:https: //doi.org/10.1016/j.actamat.2018.07.042 URL https://wwW.sciencedirect.com/science/ article/pii/S1359645418305706

[16] K. Utimula, G. I. Prayogo, K. Nakano, K. Hongo, R. Maezono, Stochastic estimations of the total number of classes for a clustering having extremely large samples to be included in the clustering engine, Advanced Theory and Simulations 4 (5) (2021) 2000301

[17] X. Chang, M. Zeng, K. Liu, L. Fu, Phase engineering of high-entropy alloys, Advanced Materials 32 (14) (2020) 1907226. arXiv:https://onlinelibrary.wiley.com/ doi/pdf/10.1002/adma.201907226 doi:https://doi. 
org/10.1002/adma.201907226.

URL https://onlinelibrary.wiley.com/doi/abs/10. 1002/adma.201907226

[18] Y. Zhang, T. T. Zuo, Z. Tang, M. C. Gao, K. A. Dahmen, P. K. Liaw, Z. P. Lu, Microstructures and properties of high-entropy alloys, Progress in Materials Science 61 (2014) 1-93.

[19] D. Miracle, O. Senkov, A critical review of high entropy alloys and related concepts, Acta Materialia 122 (2017) 448-511.

[20] L. Rogal, P. Bobrowski, F. Körmann, S. Divinski, F. Stein, B. Grabowski, Computationally-driven engineering of sublattice ordering in a hexagonal alhfsctizr high entropy alloy, Scientific Reports 7 (1) (2017) 2209.

[21] J. Ding, Q. Yu, M. Asta, R. O. Ritchie, Tunable stacking fault energies by tailoring local chemical order in crconi mediumentropy alloys Proceedings of the National Academy of Sciences 115 (36) (2018) 8919-8924. doi:10.1073/pnas. 1808660115

URL https: //www .pnas . org/content/115/36/8919

[22] Q.-J. Li, H. Sheng, E. Ma, Strengthening in multi-principal element alloys with local-chemical-order roughened dislocation pathways, Nature Communications 10 (1) (2019) 3563. doi : 10.1038/s41467-019-11464-7 URL https: //doi .org/10.1038/s41467-019-11464-7

[23] R. Zhang, S. Zhao, J. Ding, Y. Chong, T. Jia, C. Ophus, M. Asta, R. O. Ritchie, A. M. Minor, Short-range order and its impact on the crconi medium-entropy alloy, Nature 581 (7808) (2020) 283-287. doi : 10.1038/s41586-020-2275-z URL https://doi .org/10.1038/s41586-020-2275-z

[24] M. S. Lucas, G. B. Wilks, L. Mauger, J. A. Muñoz, O. N. Senkov, E. Michel, J. Horwath, S. L. Semiatin, M. B. Stone, D. L. Abernathy, E. Karapetrova, Absence of long-range chemical ordering in equimolar fecocrni Applied Physics Letters 100 (25) (2012) 251907. doi : 10.1063/1.4730327

URL https://doi.org/10.1063/1.4730327

[25] C. Niu, A. J. Zaddach, A. A. Oni, X. Sang, J. W. Hurt, J. M. LeBeau, C. C. Koch, D. L. Irving, Spin-driven ordering of cr in the equiatomic high entropy alloy nifecrco Applied Physics Letters 106 (16) (2015) 161906. doi : 10.1063/1.4918996 URL https://doi .org/10.1063/1.4918996

[26] S. Middleburgh, D. King, G. Lumpkin, M. Cortie, L. Edwards, Segregation and migration of species in the crcofeni high entropy alloy. Journal of Alloys and Compounds 599 (2014) 179-182. doi:https: //doi.org/10.1016/j.jallcom.2014.01.135 URL https://wWw.sciencedirect.com/science/ article/pii/S0925838814001819

[27] T. Fukushima, H. Katayama-Yoshida, K. Sato, M. Ogura, R. Zeller, P. H. Dederichs, Local energies and energy fluctuations - applied to the high entropy alloy crfeconi, Journal of the Physical Society of Japan 86 (11) (2017) 114704. doi: 10.7566/JPSJ .86.114704.

URL https://doi.org/10.7566/JPS] .86.114704

[28] W.-M. Choi, Y. H. Jo, S. S. Sohn, S. Lee, B.-J. Lee, Understanding the physical metallurgy of the cocrfemnni high-entropy alloy: an atomistic simulation study, npj Computational Materials 4 (1) (2018) 1. doi:10.1038/s41524-017-0060-9 URL https://doi.org/10.1038/s41524-017-0060-9

[29] J. M. Rickman, H. M. Chan, M. P. Harmer, J. A. Smeltzer, C. J. Marvel, A. Roy, G. Balasubramanian, Materials informatics for the screening of multi-principal elements and high-entropy alloys Nature Communications 10 (1) (2019) 2618.

URL https://doi .org/10.1038/s41467-019-10533-1

[30] B. Yin, W. A. Curtin, First-principles-based prediction of yield strength in the rhirpdptnicu high-entropy alloy npj
Computational Materials 5 (1) (2019) 14. doi:10.1038/ s41524-019-0151-x

URL https://doi .org/10.1038/s41524-019-0151-x

[31] M. Witman, G. Ek, S. Ling, J. Chames, S. Agarwal, J. Wong, M. D. Allendorf, M. Sahlberg, V. Stavila, Datadriven discovery and synthesis of high entropy alloy hydrides with targeted thermodynamic stability Chemistry of Materials 33 (11) (2021) 4067-4076. arXiv: https://doi.org/10.1021/acs. chemmater.1c00647. doi : 10.1021/acs. chemmater.1c00647

URL https://doi.org/10.1021/acs.chemmater. $1 \mathrm{C} 00647$

[32] H. Diao, R. Feng, K. Dahmen, P. Liaw, Fundamental deformation behavior in high-entropy alloys: An overview, Current Opinion in Solid State and Materials Science 21 (5) (2017) 252-266, concentrated Solid Solution Alloys Perspective. doi: https://doi.org/10.1016/j.cossms.2017.08.003 URL https://www.sciencedirect.com/science/ article/pii/S135902861730147X

[33] A. Bieber, F. Ducastelle, F. Gautier, G. Tréglia, P. Turchi, Electronic structure and relative stabilities of 112 and $\mathrm{d} 022$ ordered structures occuring in transition metal alloys Solid State Communications 45 (7) (1983) 585-590. doi : https: //doi.org/10.1016/0038-1098(83)90432-5 URL https://wwW.sciencedirect.com/science/ article/pii/0038109883904325

[34] E. Cabet, A. Pasturel, F. Ducastelle, A. Loiseau, $L 1_{2}-$ $d o_{22}$ competition in the pseudobinary $(\mathrm{pt}, \mathrm{rh})_{3} \mathrm{v}, \quad \mathrm{pt}_{3}(\mathrm{v}$, ti), and (pd, rh) $)_{3} \mathrm{v}$ alloys: Phase stability and electronic structure Phys. Rev. Lett. 76 (1996) 3140-3143. doi: 10.1103/PhysRevLett.76.3140.

URL https://link.aps.org/doi/10.1103/ PhysRevLett.76.3140

[35] K. Stolze, J. Tao, F. O. von Rohr, T. Kong, R. J. Cava, Sc-zr-nb-rh-pd and sc-zr-nb-ta-rh-pd high-entropy alloy superconductors on a cscl-type lattice Chemistry of Materials 30 (3) (2018) 906-914. arXiv: https://doi.org/10.1021/acs. chemmater.7b04578. doi : $10.1021 /$ acs . chemmater.7b04578 URL https://doi.org/10.1021/acs.chemmater. $7 \mathrm{~b} 04578$

[36] F. He, D. Chen, B. Han, Q. Wu, Z. Wang, S. Wei, D. Wei, J. Wang, C. Liu, J. jung Kai, Design of d022 superlattice with superior strengthening effect in high entropy alloys Acta Materialia 167 (2019) 275-286. doi:https: //doi.org/10.1016/j.actamat.2019.01.048 URL https://www.sciencedirect.com/science/ article/pii/S1359645419300631

[37] N. Zhou, S. Jiang, T. Huang, M. Qin, T. Hu, J. Luo, Single-phase high-entropy intermetallic compounds (heics): bridging high-entropy alloys and ceramics, Science Bulletin 64 (12) (2019) 856-864, sPECIAL TOPIC: Electromagnetic Metasurfaces: from Concept to Applications. doi:https://doi.org/10.1016/j.scib.2019.05.007 URL https://wwW.sciencedirect.com/science/ article/pii/S2095927319302919

[38] J. Zhang, J. Yan, S. Calder, Q. Zheng, M. A. McGuire, D. L. Abernathy, Y. Ren, S. H. Lapidus, K. Page, H. Zheng, J. W. Freeland, J. D. Budai, R. P. Hermann, Long-range antiferromagnetic order in a rocksalt high entropy oxide Chemistry of Materials 31 (10) (2019) 3705-3711. arXiv: https://doi.org/10.1021/acs . chemmater.9b00624. doi : 10.1021/acs. chemmater. 9b00624 URL https://doi.org/10.1021/acs.chemmater. 
9b00624

[39] S. Guo, C. Ng, J. Lu, C. T. Liu, Effect of valence electron concentration on stability of fcc or bcc phase in high entropy alloys, Journal of Applied Physics 109 (10) (2011) 103505. doi: $10.1063 / 1.3587228$

URL https://doi .org/10.1063/1.3587228

[40] S. GUO, C. LIU, Phase stability in high entropy alloys: Formation of solid-solution phase or amorphous phase Progress in Natural Science: Materials International 21 (6) (2011) 433-446. doi:https: //doi.org/10.1016/S1002-0071(12)60080-X URL https://www.sciencedirect.com/science/ article/pii/S100200711260080X

[41] F. Tian, L. K. Varga, N. Chen, J. Shen, L. Vitos, Empirical design of single phase high-entropy alloys with high hardness Intermetallics 58 (2015) 1-6. doi:https: //doi.org/10.1016/j.intermet.2014.10.010 URL https://wWW.sciencedirect.com/science/ article/pii/S0966979514002775

[42] Z. Leong, J. S. Wróbel, S. L. Dudarev, R. Goodall, I. Todd, D. Nguyen-Manh, The effect of electronic structure on the phases present in high entropy alloys. Scientific Reports 7 (1) (2017) 39803. doi : 10.1038/srep39803

URL https://doi.org/10.1038/srep39803

[43] A. Zunger, S.-H. Wei, L. G. Ferreira, J. E. Bernard, Special quasirandom structures Phys. Rev. Lett. 65 (1990) 353-356. doi:10.1103/PhysRevLett.65.353 URL https://link.aps.org/doi/10.1103/ PhysRevLett.65.353

[44] C. Niu, C. R. LaRosa, J. Miao, M. J. Mills, M. Ghazisaeidi, Magnetically-driven phase transformation strengthening in high entropy alloys Nature Communications 9 (1) (2018) 1363. doi : $10.1038 / \mathrm{s} 41467-018-03846-0$

URL https://doi .org/10.1038/s41467-018-03846-0

[45] J. M. Cowley, Short- and long-range order parameters in disordered solid solutions, Phys. Rev. 120 (1960) 1648-1657. doi : 10.1103/PhysRev. 120.1648

URL https://link.aps.org/doi/10.1103/PhysRev. 120.1648

[46] J. M. Cowley, Short-range order and long-range order parameters Phys. Rev. 138 (1965) A1384-A1389. doi: 10.1103/PhysRev. 138.A1384

URL https://link.aps.org/doi/10.1103/PhysRev. 138.A1384

[47] N. Metropolis, A. W. Rosenbluth, M. N. Rosenbluth, A. H. Teller, E. Teller, Equation of state calculations by fast computing machines The Journal of Chemical Physics 21 (6) (1953) 1087-1092. doi: 10.1063/1.1699114

URL https://doi.org/10.1063/1.1699114

[48] K. Momma, F. Izumi, VESTA3 for three-dimensional visualization of crystal, volumetric and morphology data Journal of Applied Crystallography 44 (6) (2011) 1272-1276. doi : 10.1107/S0021889811038970

URL https://doi .org/10.1107/S0021889811038970

[49] W. Kohn, L. J. Sham, Self-consistent equations including exchange and correlation effects Phys. Rev. 140 (1965) A1133-A1138. doi:10.1103/PhysRev. 140.A1133 URL https://link.aps.org/doi/10.1103/PhysRev. 140.A1133

[50] G. Kresse, J. Furthmüller, Efficiency of ab-initio total energy calculations for metals and semiconductors using a plane-wave basis set Computational Materials Science 6 (1) (1996) 15-50. doi:https: //doi.org/10.1016/0927-0256(96)00008-0
URL https://wwW.sciencedirect.com/science/ article/pii/0927025696000080

[51] G. Kresse, J. Furthmüller, Efficient iterative schemes for $a b$ initio total-energy calculations using a planewave basis set Phys. Rev. B 54 (1996) 11169-11186. doi: 10.1103/PhysRevB.54.11169

URL https://link.aps.org/doi/10.1103/PhysRevB. 54.11169

[52] J. P. Perdew, A. Ruzsinszky, G. I. Csonka, O. A. Vydrov, G. E. Scuseria, L. A. Constantin, X. Zhou, K. Burke, Restoring the density-gradient expansion for exchange in solids and surfaces Phys. Rev. Lett. 100 (2008) 136406. doi:10.1103/PhysRevLett.100.136406.

URL https://link.aps.org/doi/10.1103/ PhysRevLett. 100.136406

[53] P. E. Blöchl, Projector augmented-wave method Phys. Rev. B 50 (1994) 17953-17979. doi:10.1103/PhysRevB.50. 17953.

URL https://link.aps.org/doi/10.1103/PhysRevB. 50.17953

[54] G. Kresse, D. Joubert, From ultrasoft pseudopotentials to the projector augmented-wave method, Phys. Rev. B 59 (1999) 1758-1775. doi:10.1103/PhysRevB.59.1758.

URL https://link.aps.org/doi/10.1103/PhysRevB. 59.1758

[55] H. J. Monkhorst, J. D. Pack, Special points for brillouinzone integrations Phys. Rev. B 13 (1976) 5188-5192. doi : 10.1103/PhysRevB. 13.5188

URL https://link.aps.org/doi/10.1103/PhysRevB. 13.5188

[56] J. E. Saal, S. Kirklin, M. Aykol, B. Meredig, C. Wolverton, Materials design and discovery with high-throughput density functional theory: The open quantum materials database (oqmd), JOM 65 (11) (2013) 1501-1509. doi:10.1007/ s11837-013-0755-4 URL https://doi .org/10.1007/s11837-013-0755-4

[57] S. Kirklin, J. E. Saal, B. Meredig, A. Thompson, J. W. Doak, M. Aykol, S. Rühl, C. Wolverton, The open quantum materials database (oqmd): assessing the accuracy of $\mathrm{dft}$ formation energies npj Computational Materials 1 (1) (2015) 15010. doi : 10.1038/npjcompumats.2015.10 URL https://doi.org/10.1038/npjcompumats.2015. 10

[58] W. Zhou, R. Sahara, K. Tsuchiya, First-principles study of the phase stability and elastic properties of ti-x alloys $(\mathrm{x}=\mathrm{mo}, \mathrm{nb}, \mathrm{al}, \mathrm{sn}, \mathrm{zr}, \mathrm{fe}, \mathrm{co}$, and o) Journal of Alloys and Compounds 727 (2017) 579-595. doi:https: //doi.org/10.1016/j.jallcom.2017.08.128 URL https://www.sciencedirect.com/science/ article/pii/S0925838817328578

[59] C. Niu, Y. Rao, W. Windl, M. Ghazisaeidi, Multi-cell monte carlo method for phase prediction npj Computational Materials 5 (1) (2019) 120. doi : 10 . 1038/s41524-019-0259-z URL https://doi .org/10.1038/s41524-019-0259-z

[60] A. van de Walle, G. Ceder, U. V. Waghmare, First-principles computation of the vibrational entropy of ordered and disordered $\mathrm{ni}_{3} \mathrm{Al}$, Phys. Rev. Lett. 80 (1998) 4911-4914. doi: 10.1103/PhysRevLett.80.4911. URL https://link.aps.org/doi/10.1103/ PhysRevLett.80.4911

[61] D. Ma, B. Grabowski, F. Körmann, J. Neugebauer, D. Raabe, $\mathrm{Ab}$ initio thermodynamics of the cocrfemnni high entropy alloy: Importance of entropy contributions beyond the configurational one Acta Materialia 100 (2015) 90-97. doi : https: 
//doi.org/10.1016/j.actamat.2015.08.050 URL https://www.sciencedirect.com/science/ article/pii/S1359645415006278

[62] J.-W. Yeh, Alloy design strategies and future trends in highentropy alloys, JOM 65 (12) (2013) 1759-1771. doi:10. 1007/s11837-013-0761-6

URL https://doi .org/10.1007/s11837-013-0761-6

[63] M. C. Troparevsky, J. R. Morris, P. R. C. Kent, A. R. Lupini, G. M. Stocks, Criteria for predicting the formation of singlephase high-entropy alloys Phys. Rev. X 5 (2015) 011041. doi : 10.1103/PhysRevX.5.011041

URL https://link.aps.org/doi/10.1103/PhysRevX. 5.011041

[64] W. Kucza, High entropy alloys and thermodynamically derived high stability alloys; a compositional comparative study of all 3-7 element systems composed of ten 3d metals at $1273 \mathrm{k}$ Journal of Alloys and Compounds 894 (2022) 162443. doi: https://doi.org/10.1016/j.jallcom.2021.162443 URL https://wWW.sciencedirect.com/science/ article/pii/S0925838821038536

[65] A. Takeuchi, A. Inoue, Classification of bulk metallic glasses by atomic size difference, heat of mixing and period of constituent elements and its application to characterization of the main alloying element, MATERIALS TRANSACTIONS 46 (12) (2005) 2817-2829. doi:10.2320/matertrans .46.2817

[66] T. Zuo, M. C. Gao, L. Ouyang, X. Yang, Y. Cheng, R. Feng, S. Chen, P. K. Liaw, J. A. Hawk, Y. Zhang, Tailoring magnetic behavior of cofemnnix (x = al, cr, ga, and sn) high entropy alloys by metal doping Acta Materialia 130 (2017) 10-18. doi : https://doi.org/10.1016/j.actamat.2017.03.013 URL https://www.sciencedirect.com/science/ article/pii/S1359645417302008

[67] M. Fedorov, J. S. Wróbel, A. Fernández-Caballero, K. J. Kurzydłowski, D. Nguyen-Manh, Phase stability and magnetic properties in fcc fe-cr-mn-ni alloys from firstprinciples modeling, Phys. Rev. B 101 (2020) 174416. doi: 10.1103/PhysRevB.101.174416

URL https://link.aps.org/doi/10.1103/PhysRevB. 101.174416

[68] B. Schönfeld, C. R. Sax, J. Zemp, M. Engelke, P. Boesecke, T. Kresse, T. Boll, T. Al-Kassab, O. E. Peil, A. V. Ruban, Local order in cr-fe-co-ni: Experiment and electronic structure calculations, Phys. Rev. B 99 (2019) 014206. doi:10.1103/PhysRevB.99.014206

URL https://link.aps.org/doi/10.1103/PhysRevB. 99.014206

[69] M. C. Gao, D. E. Alman, Searching for next single-phase high-entropy alloy compositions Entropy 15 (10) (2013) 45044519. doi: $10.3390 / \mathrm{e} 15104504$

URL https: //www .mdpi . com/1099-4300/15/10/4504

[70] W. Xiong, G. B. Olson, Cybermaterials: materials by design and accelerated insertion of materials npj Computational Materials 2 (1) (2016) 15009. doi:10.1038/npjcompumats. 2015.9 .

URL https://doi .org/10.1038/npjcompumats. 2015.9

[71] C. Han, Q. Fang, Y. Shi, S. B. Tor, C. K. Chua, K. Zhou, Recent advances on high-entropy alloys for 3d printing Advanced Materials 32 (26) (2020) 1903855. arXiv:https://onlinelibrary.wiley. com/doi/pdf/10.1002/adma.201903855 doi:https: //doi.org/10.1002/adma.201903855.

URL https://onlinelibrary.wiley.com/doi/abs/10. 1002/adma. 201903855

[72] C. Kenel, N. P. M. Casati, D. C. Dunand, 3d ink-extrusion additive manufacturing of cocrfeni high-entropy alloy microlattices Nature Communications 10 (1) (2019) 904. doi: 10.1038/s41467-019-08763-4

URL https://doi .org/10.1038/s41467-019-08763-4

[73] N. Saunders, A. Miodownik, CALPHAD (Calculation of Phase Diagrams): A Comprehensive Guide ISSN, Elsevier Science, 1998.

URL ROnQVSNLZUAC

[74] A. T. Hanindriyo, S. Sridar, K. H. Kumar, K. Hongo, R. Maezono, $\mathrm{Ab}$ initio thermodynamic properties of certain compounds in nd-fe-b system Computational Materials Science 180 (2020) 109696. doi:https: //doi.org/10.1016/j.commatsci.2020.109696 URL https://wWw.sciencedirect.com/science/ article/pii/S0927025620301877

[75] S. Bhattacharyya, R. Sahara, K. Ohno, A first-principles phase field method for quantitatively predicting multi-composition phase separation without thermodynamic empirical parameter. Nature Communications 10 (1) (2019) 3451. doi:10.1038/ s41467-019-11248-z

URL https://doi.org/10.1038/s41467-019-11248-z

[76] G. Anand, A. P. Wynn, C. M. Handley, C. L. Freeman, Phase stability and distortion in high-entropy oxides Acta Materialia 146 (2018) 119-125. doi:https: //doi.org/10.1016/j.actamat.2017.12.037 URL https://www.sciencedirect.com/science/ article/pii/S1359645417310492

[77] A. Sarkar, Q. Wang, A. Schiele, M. R. Chellali, S. S. Bhattacharya, D. Wang, T. Brezesinski, H. Hahn, L. Velasco, B. Breitung, High-entropy oxides: Fundamental aspects and electrochemical properties Advanced Materials 31 (26) (2019) 1806236. arXiv:https://onlinelibrary.wiley.com/ doi/pdf/10.1002/adma.201806236 doi:https://doi. org/10.1002/adma.201806236

URL https://onlinelibrary.wiley.com/doi/abs/10. 1002/adma. 201806236

[78] T. J. Harrington, J. Gild, P. Sarker, C. Toher, C. M. Rost, O. F. Dippo, C. McElfresh, K. Kaufmann, E. Marin, L. Borowski, P. E. Hopkins, J. Luo, S. Curtarolo, D. W. Brenner, K. S. Vecchio, Phase stability and mechanical properties of novel high entropy transition metal carbides, Acta Materialia 166 (2019) 271-280. doi:https: //doi.org/10.1016/j.actamat.2018.12.054

URL https://wwW.sciencedirect.com/science/ article/pii/S135964541831005X

[79] B. Ye, T. Wen, M. C. Nguyen, L. Hao, C.-Z. Wang, Y. Chu, First-principles study, fabrication and characterization of $(\mathrm{zr} 0.25 \mathrm{nb} 0.25 \mathrm{ti} 0.25 \mathrm{v} 0.25) \mathrm{c}$ high-entropy ceramics Acta Materialia 170 (2019) 15-23. doi:https: //doi.org/10.1016/j.actamat.2019.03.021 URL https://wwW.sciencedirect.com/science/ article/pii/S1359645419301648

[80] C. Oses, C. Toher, S. Curtarolo, High-entropy ceramics Nature Reviews Materials 5 (4) (2020) 295-309. doi:10.1038/ s41578-019-0170-8

URL https://doi .org/10.1038/s41578-019-0170-8

[81] K. Kaufmann, D. Maryanovsky, W. M. Mellor, C. Zhu, A. S. Rosengarten, T. J. Harrington, C. Oses, C. Toher, S. Curtarolo, K. S. Vecchio, Discovery of high-entropy ceramics via machine learning npj Computational Materials 6 (1) (2020) 42. doi: 10. $1038 / \mathrm{s} 41524-020-0317-6$

URL https://doi .org/10.1038/s41524-020-0317-6

[82] S. J. McCormack, A. Navrotsky, Thermodynamics of high 
Table of Contents

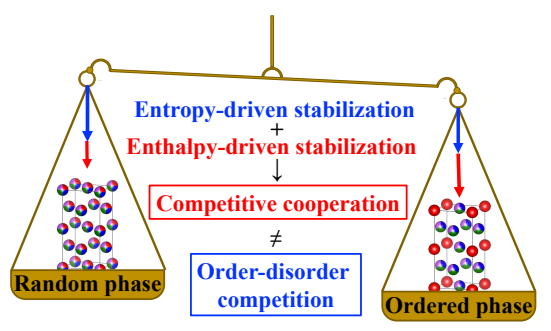

Phase stability of 3d-transition metal high-entropy alloys (HEAs) is realized by an order-disorder "competitive cooperation," rather than a usual order-disorder competition. The former was newly proposed in this study, while the latter has already been accepted in the HEA community.

entropy oxides, Acta Materialia 202 (2021) 1-21. doi : https: //doi.org/10.1016/j.actamat.2020.10.043 URL https://www.sciencedirect.com/science/ article/pii/S1359645420308417

[83] M. D. Hossain, T. Borman, C. Oses, M. Esters, C. To- her, L. Feng, A. Kumar, W. G. Fahrenholtz, S. Curtarolo, D. Brenner, J. M. LeBeau, J.-P. Maria, Entropy landscaping of high-entropy carbides Advanced Materials n/a (n/a) 2102904. arXiv:https://onlinelibrary.wiley.com/ doi/pdf/10.1002/adma.202102904 doi:https://doi. org/10.1002/adma.202102904

URL https://onlinelibrary.wiley.com/doi/abs/10. 1002/adma. 202102904

[84] O. N. Senkov, J. D. Miller, D. B. Miracle, C. Woodward, Accelerated exploration of multi-principal element alloys with solid solution phases Nature Communications 6 (1) (2015) 6529. doi: $10.1038 /$ ncomms7529.

URL https://doi .org/10.1038/ncomms7529

[85] M. Zheng, W. Ding, W. Cao, S. Hu, Q. Huang, A quick screening approach for design of multi-principal element alloy with solid solution phase Materials \& Design 179 (2019) 107882. doi:https://doi.org/10.1016/j.matdes. 2019.107882

URL https://www.sciencedirect.com/science/ article/pii/SQ26412751930320X

[86] A. M. Manzoni, U. Glatzel, New multiphase compositionally complex alloys driven by the high entropy alloy approach Materials Characterization 147 (2019) 512-532. doi : https: //doi.org/10.1016/j.matchar.2018.06.036 URL https://wwW.sciencedirect.com/science/ article/pii/S1044580318311094 

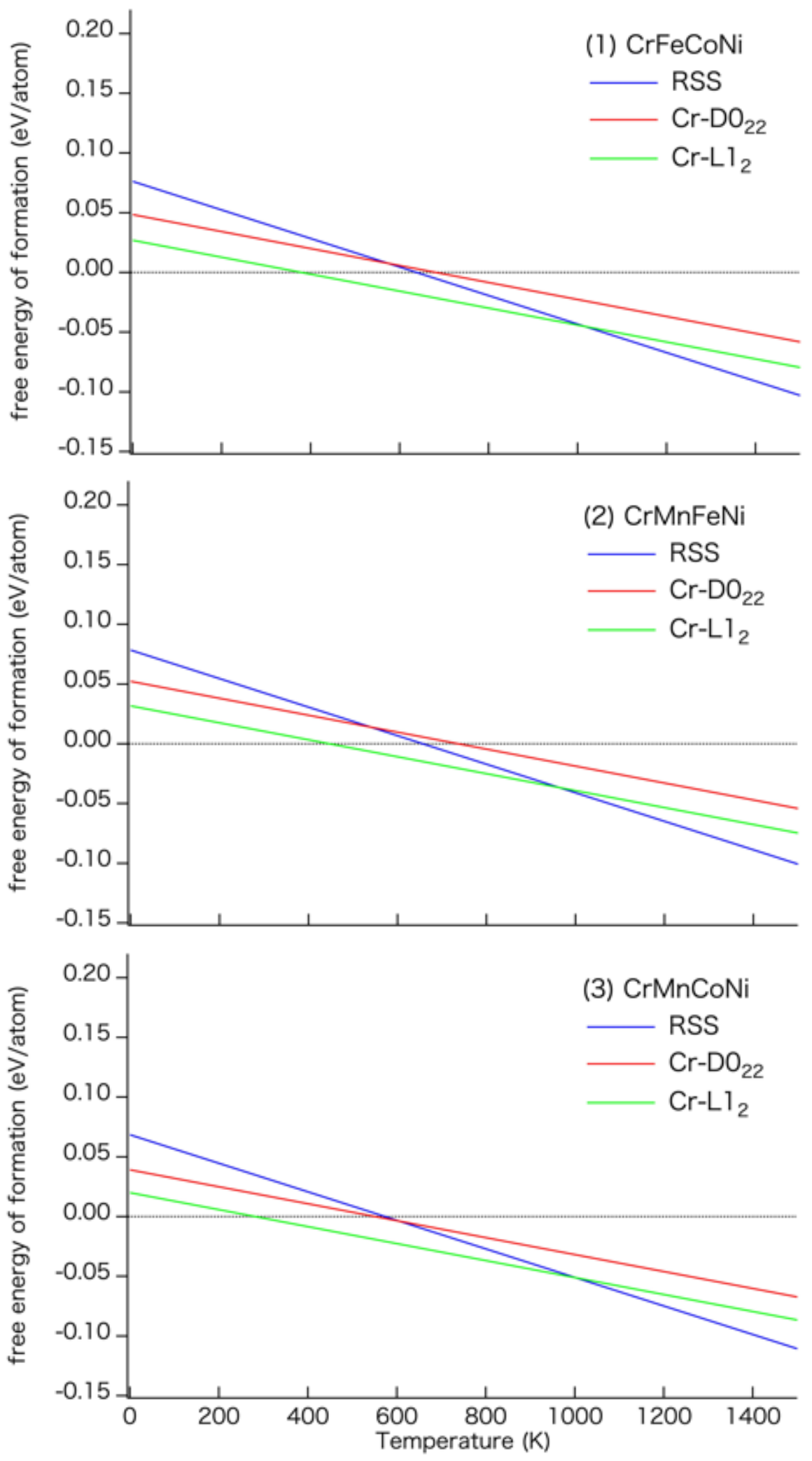

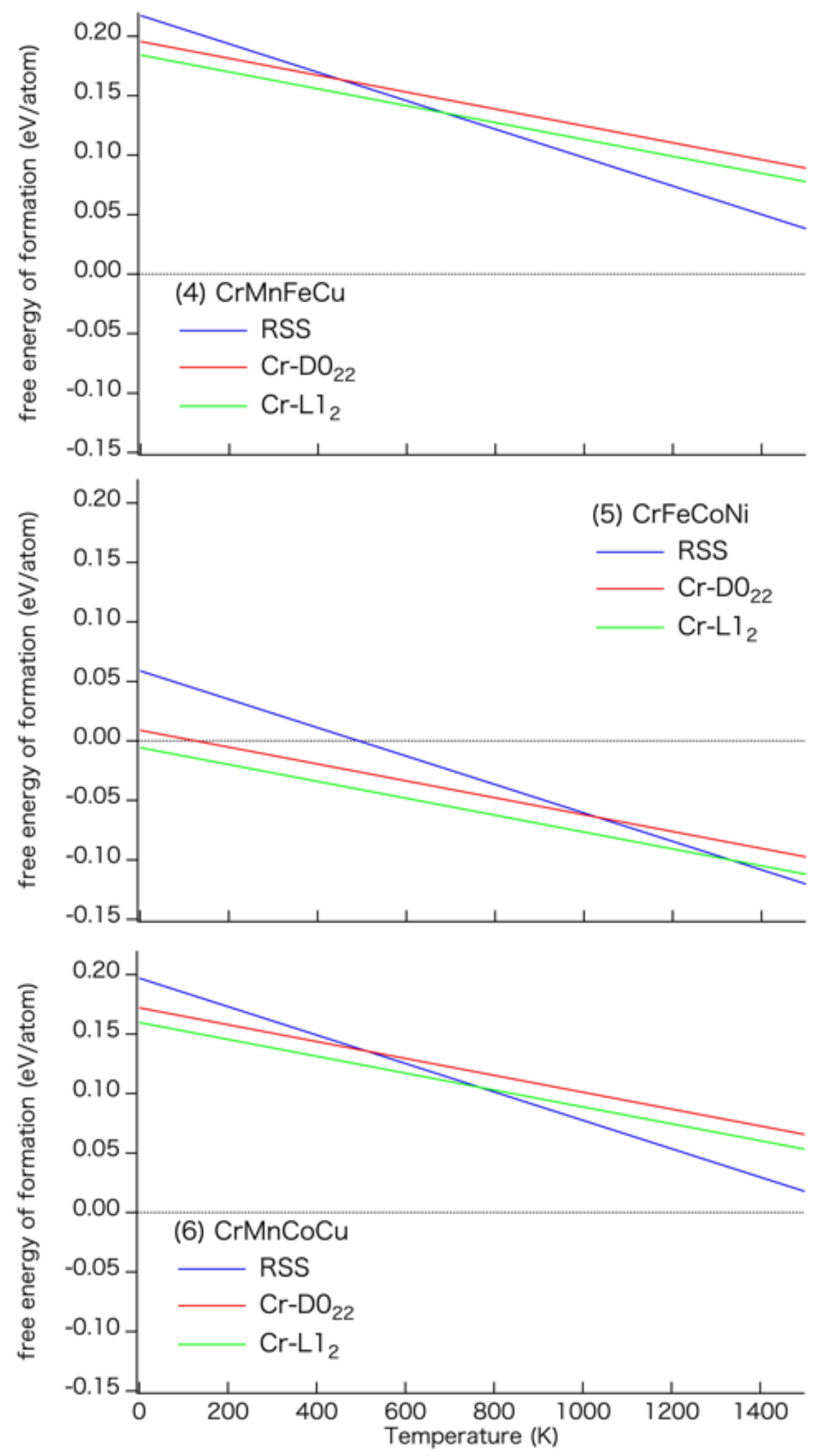

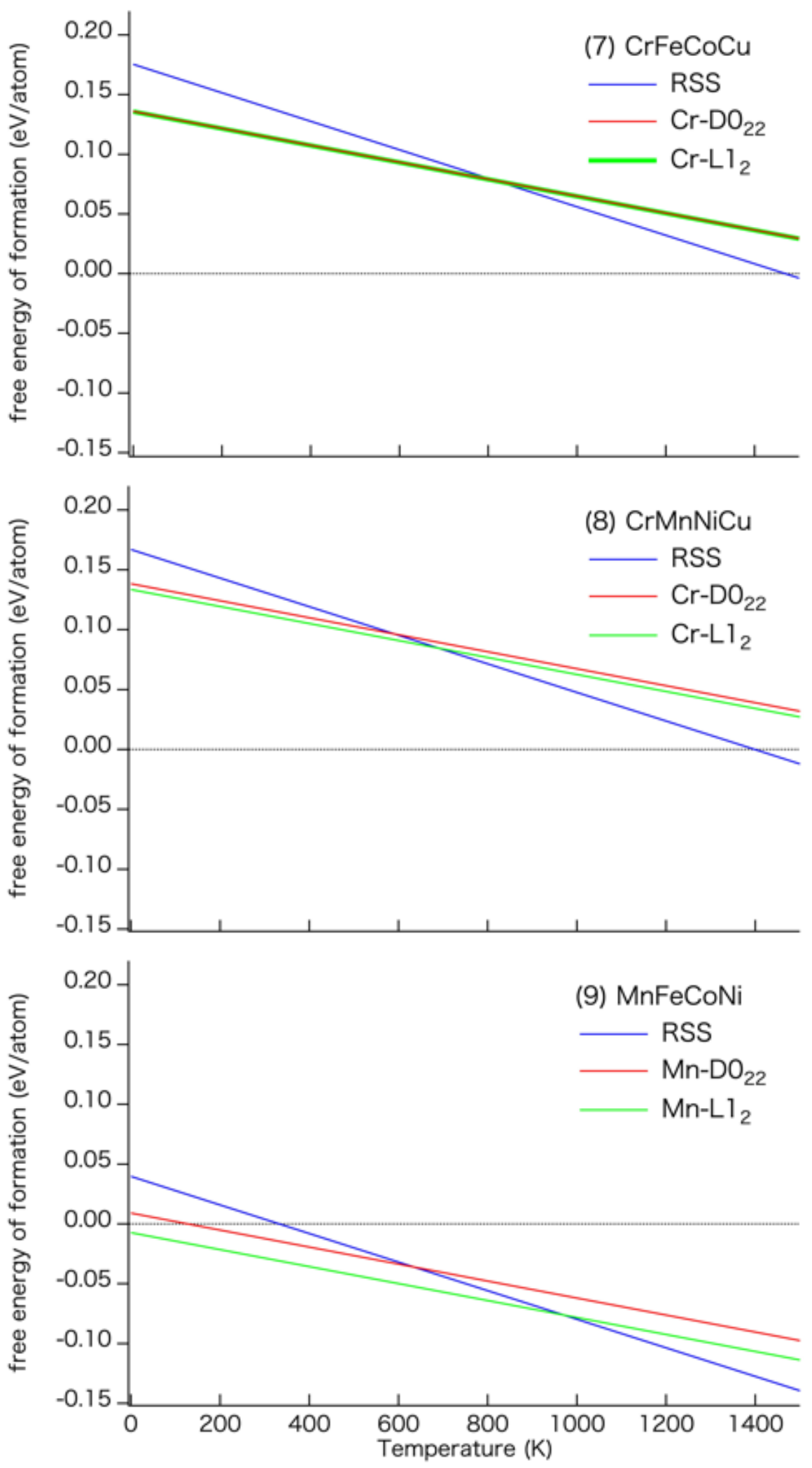

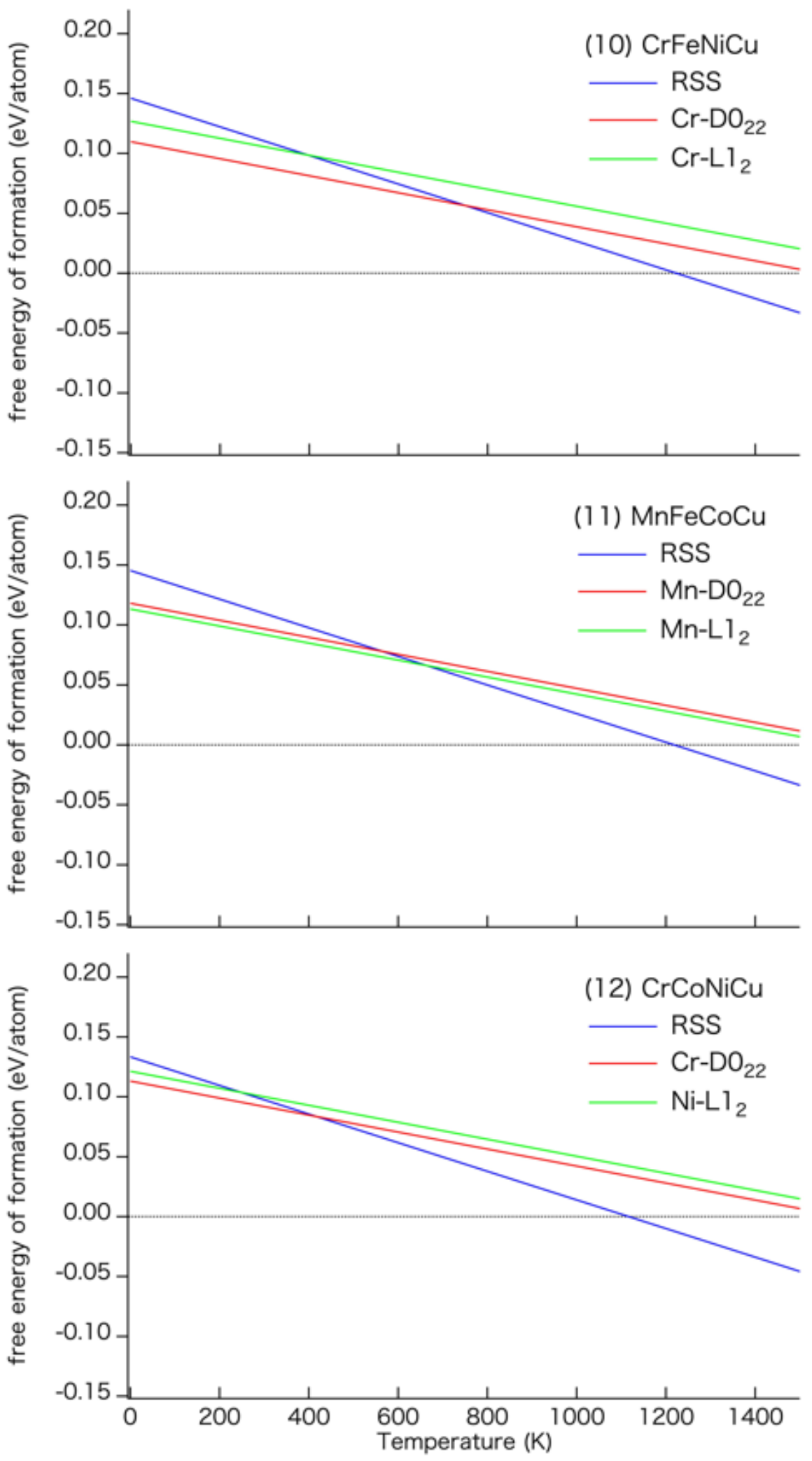

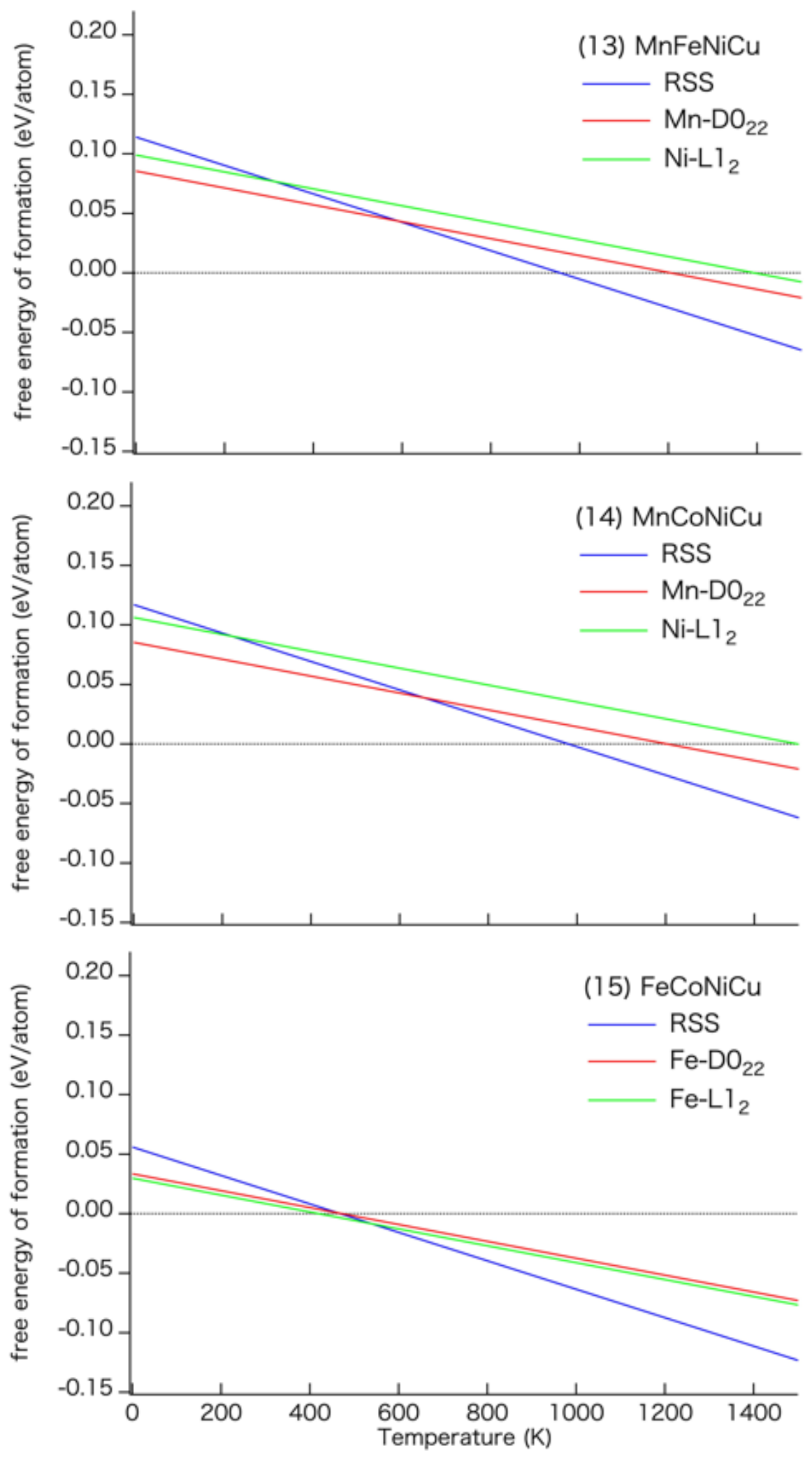

FIG. S-1: Formation free energy as a function of temperature for 15 kinds of quaternary alloys, which are listed at Table I. Blue, red and green lines indicate $\mathrm{RSS}, \mathrm{DO}_{22}$, and $\mathrm{L}_{2}$ ordered phases, respectively. 

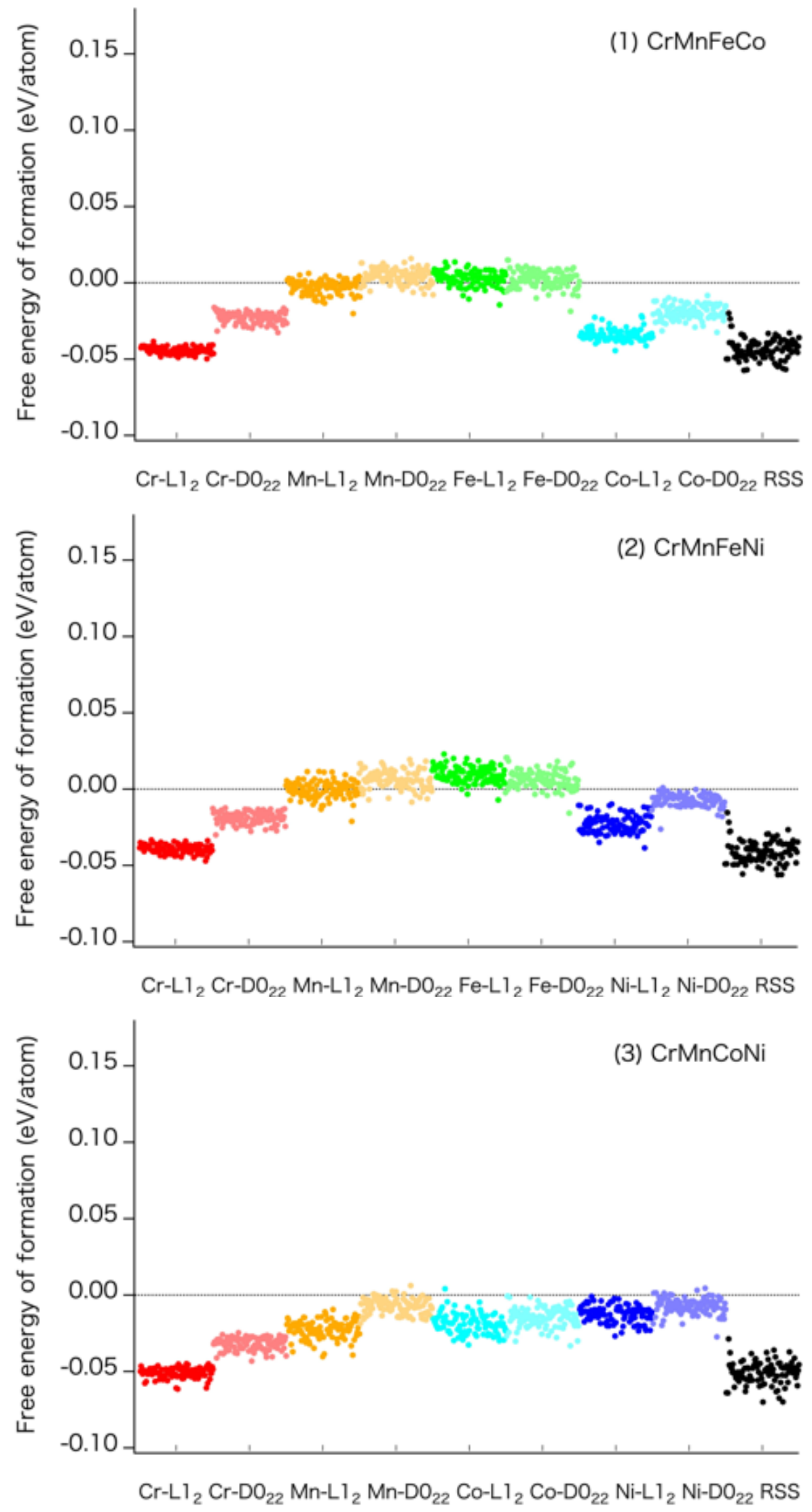

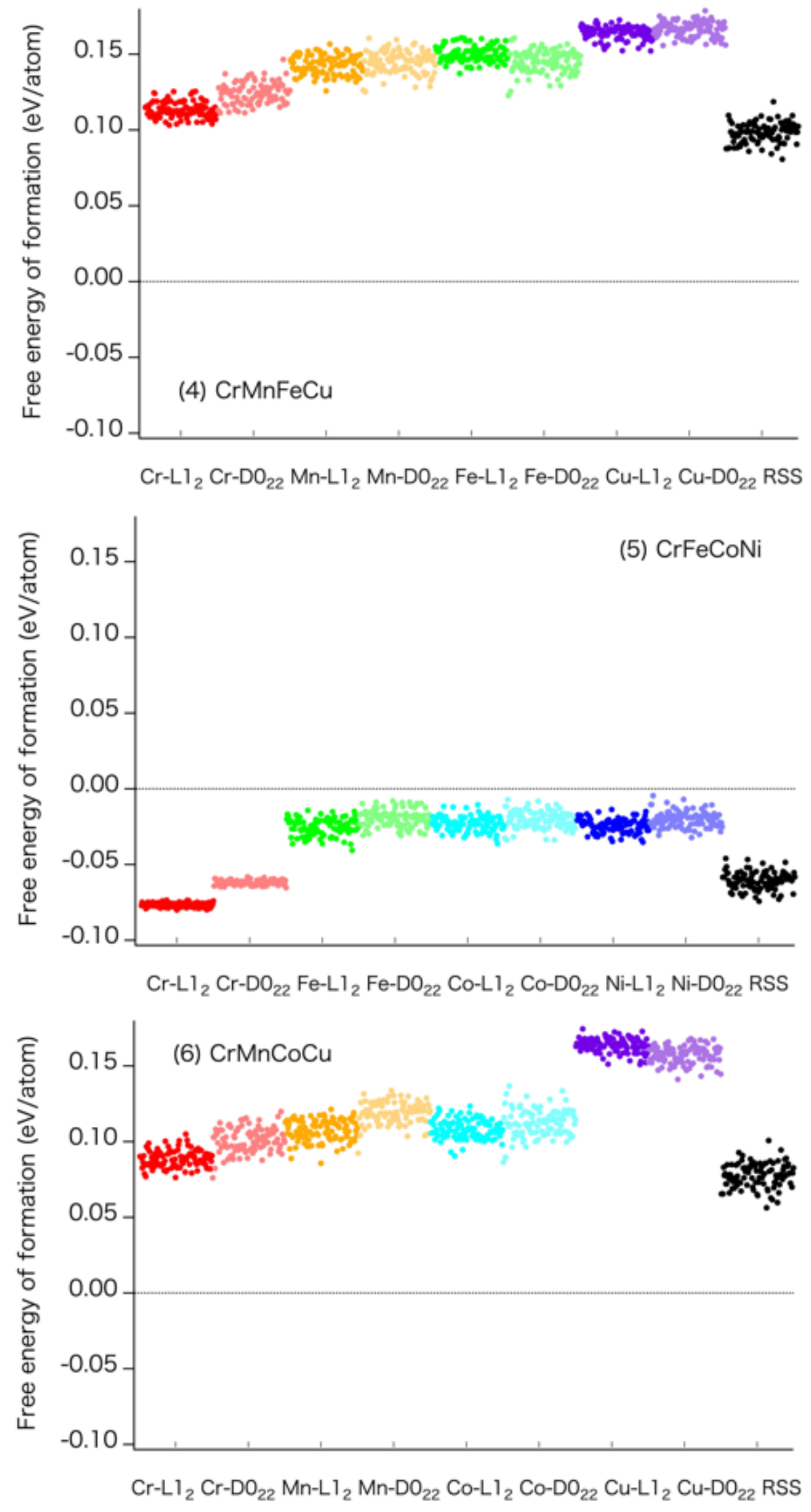

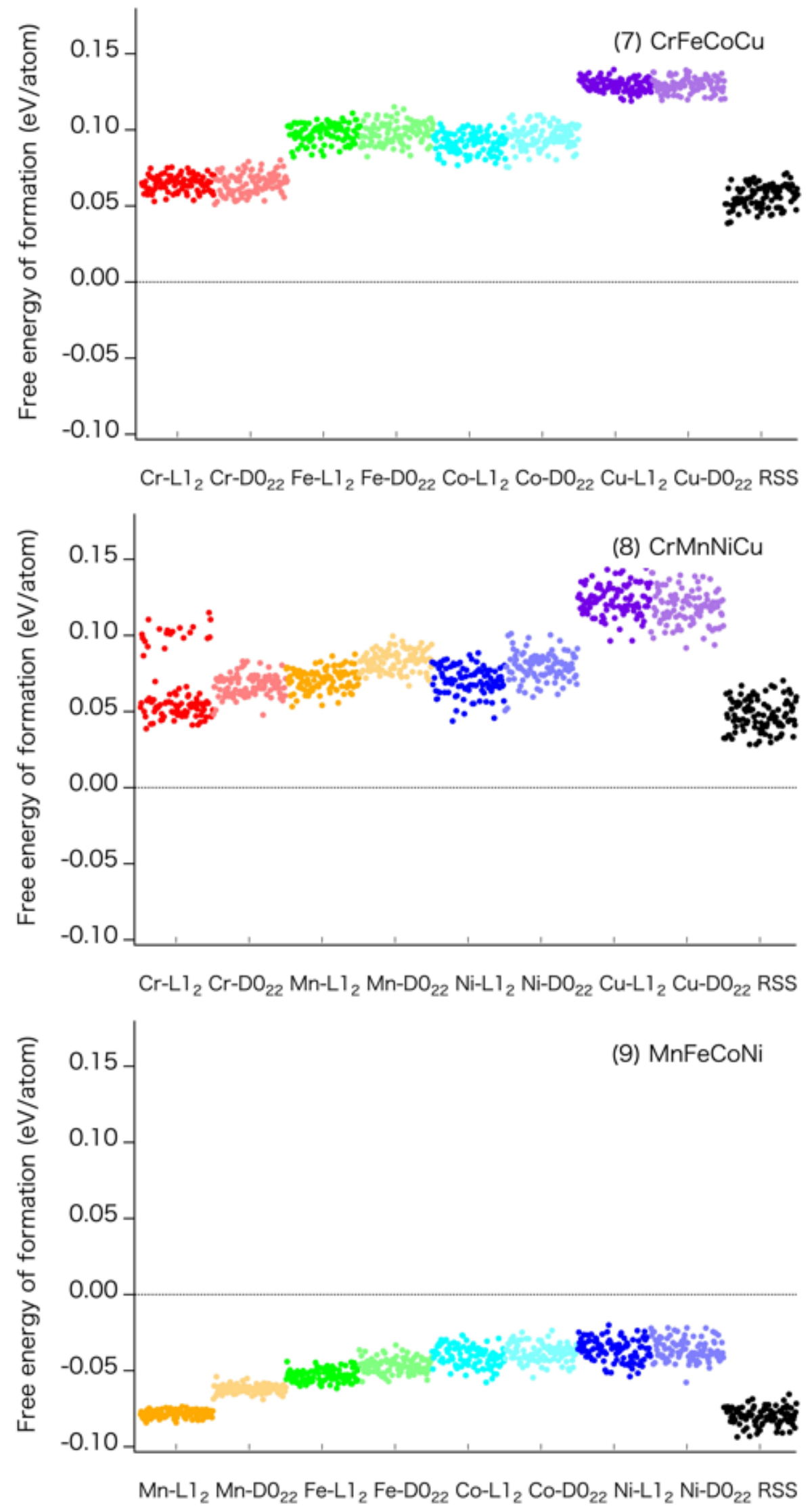

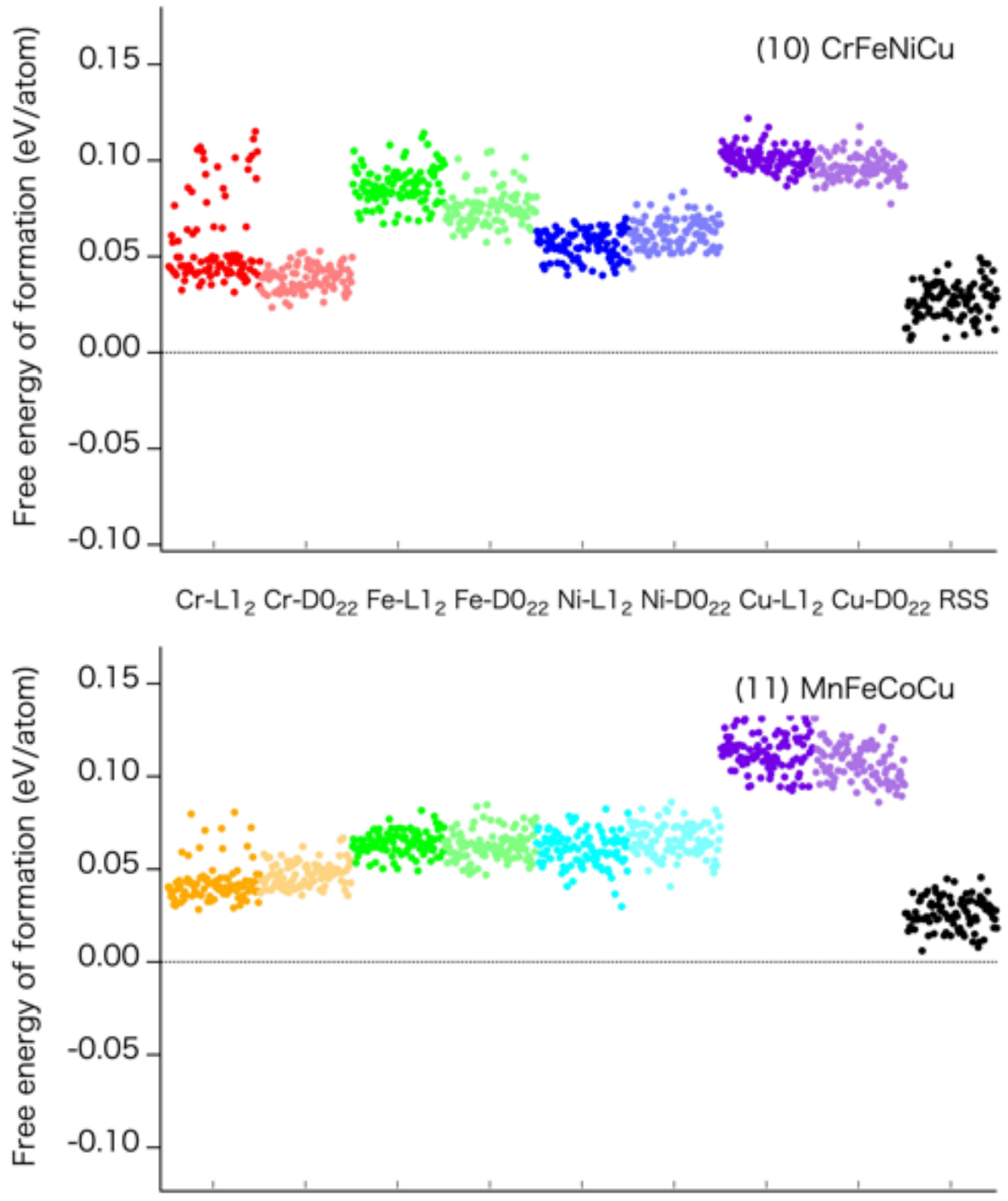

$\mathrm{Mn}-\mathrm{Ll}_{2} \mathrm{Mn}-\mathrm{DO}_{22} \mathrm{Fe}-\mathrm{Ll}_{2} \mathrm{Fe}-\mathrm{DO} \mathrm{2}_{22} \mathrm{Co}-\mathrm{Ll}_{2} \mathrm{Co}_{\mathrm{DO}} \mathrm{Cu}_{22} \mathrm{Cl} \mathrm{l}_{2} \mathrm{Cu}-\mathrm{DO}_{22} \mathrm{RSS}$

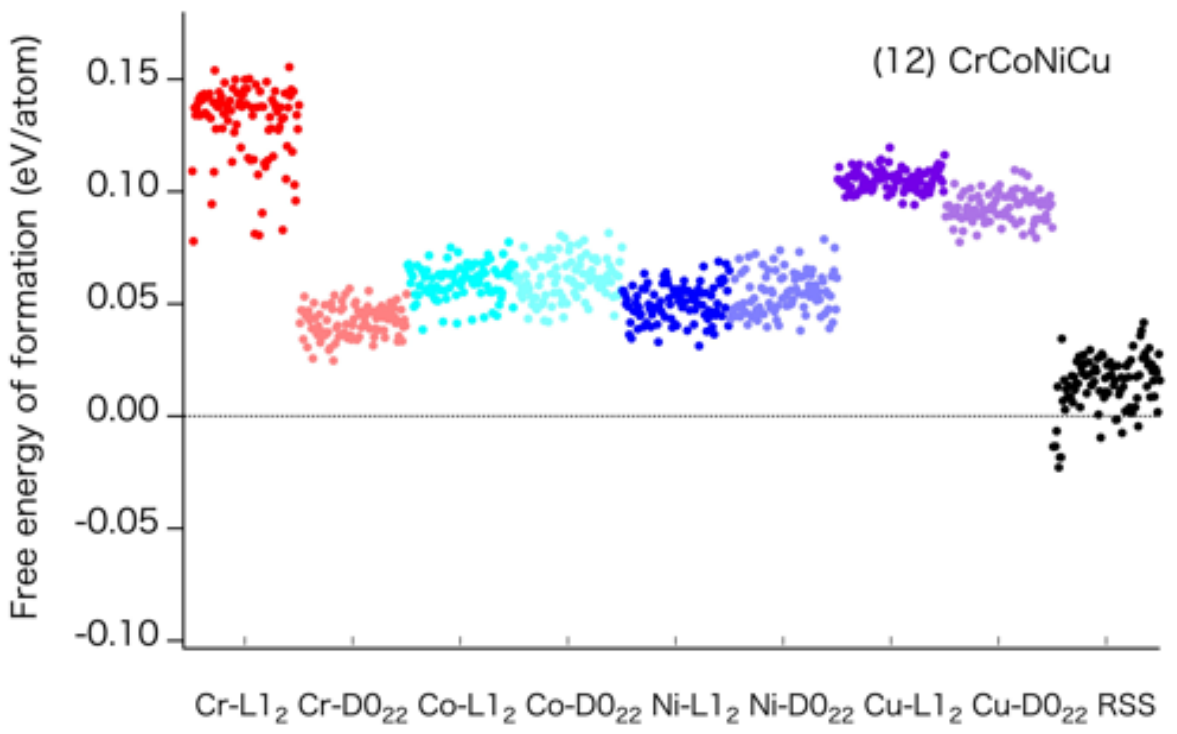



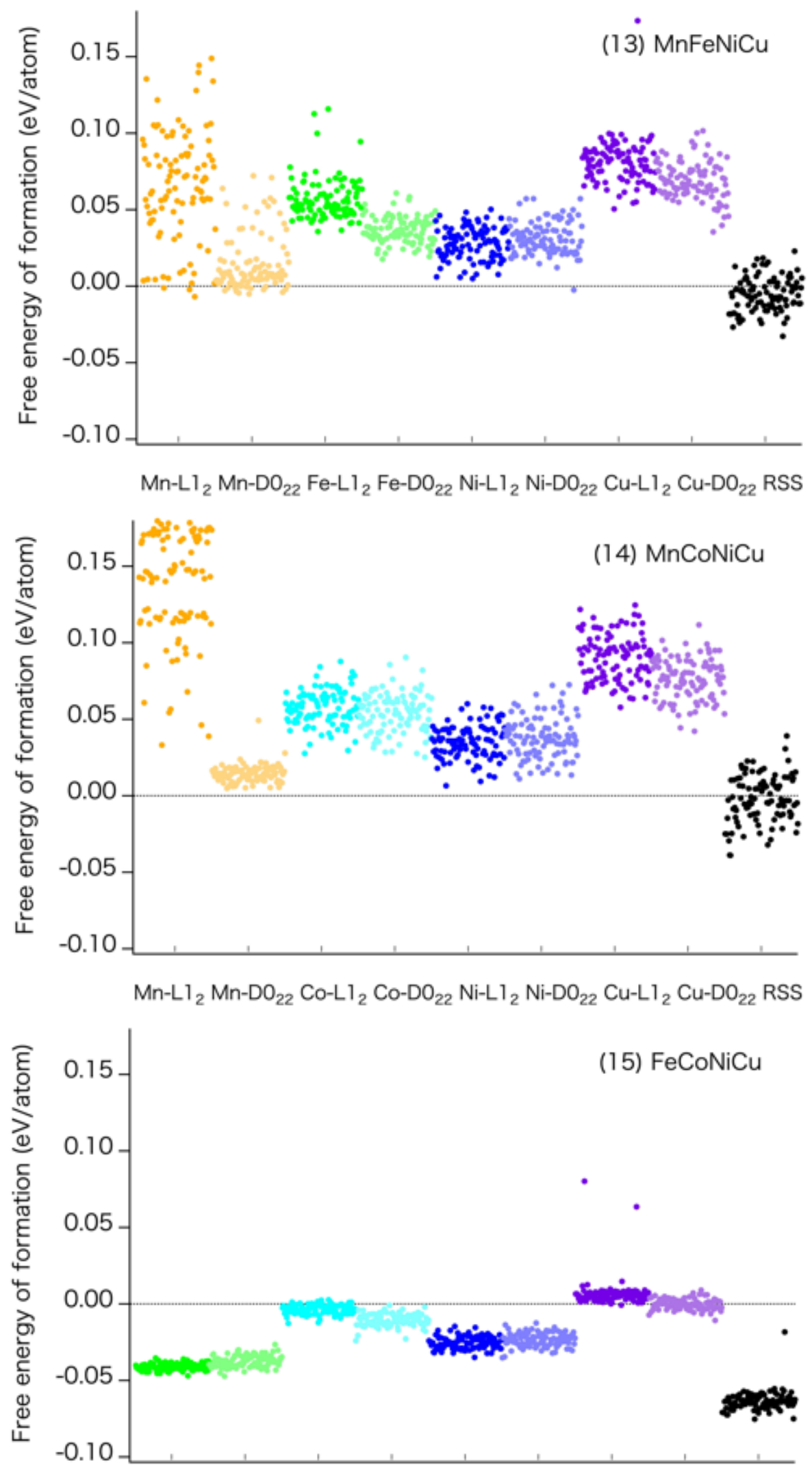

$\mathrm{Fe}-\mathrm{Ll}_{2} \mathrm{Fe}-\mathrm{DO}_{22} \mathrm{Co}-\mathrm{Ll}_{2} \mathrm{Co}_{2} \mathrm{DO}_{22} \mathrm{Ni}-\mathrm{Ll}{ }_{2} \mathrm{Ni}-\mathrm{DO}_{22} \mathrm{Cu}_{-} \mathrm{Ll}_{2} \mathrm{Cu}-\mathrm{DO}_{22} \mathrm{RSS}$

FIG. S-2: Distribution of the formation free energies at 1,000 $\mathrm{K}$ for 15 kinds of quaternary alloys, which are listed at Table I using 100 samples. Red, orange, green, cyan, blue and purple indicate $\mathrm{Cr}, \mathrm{Mn}, \mathrm{Fe}, \mathrm{Co}, \mathrm{Ni}$, and $\mathrm{Cu}$, respectively. Dark and light colors indicate $\mathrm{X}-\mathrm{L}_{2}, \mathrm{X}-\mathrm{D0}_{22}$ phases, respectively. Black indicates RSS phase. 